\title{
The dynamical balance, transport and circulation of the Antarctic Circumpolar Current
}

\author{
DIRK OLBERS ${ }^{1 *}$, DANIEL BOROWSKI ${ }^{1}$, CHRISTOPH VÖLKER ${ }^{1}$ and JÖRG-OLAF WOLFF ${ }^{2}$ \\ ${ }^{1}$ Alfred Wegener Institute for Polar and Marine Research, D-27515 Bremerhaven, Germany \\ ${ }^{2}$ ICBM, University of Oldenburg, D-26111 Oldenburg, Germany \\ *corresponding author: dolbers@awi-bremerhaven.de
}

\begin{abstract}
The physical elements of the circulation of the Antarctic Circumpolar Current (ACC) are reviewed. A picture of the circulation is sketched by means of recent observations from the WOCE decade. We present and discuss the role of forcing functions (wind stress, surface buoyancy flux) in the dynamical balance of the flow and in the meridional circulation and study their relation to the ACC transport. The physics of form stress at tilted isopycnals and at the ocean bottom are elucidated as central mechanisms in the momentum balance. We explain the failure of the Sverdrup balance in the ACC circulation and highlight the role of geostrophic contours in the balance of vorticity. Emphasis is on the interrelation of the zonal momentum balance and the meridional circulation, the importance of diapycnal mixing and eddy processes. Finally, new model concepts are described: a model of the ACC transport dependence on wind stress and buoyancy flux, based on linear wave theory; and a model of the meridional overturning and the mean density structure of the Southern Ocean, based on zonally averaged dynamics and thermodynamics with eddy parametrization.
\end{abstract}

Received 22 December 2003, accepted 1 September 2004

Key words: ACC, Southern Ocean, form stress, meridional overturning, transient and standing eddies

\section{Introduction}

Roughly $75 \%$ of the world ocean volume has temperatures below $4{ }^{\circ} \mathrm{C}$, connected (at polar latitudes) with only $2 \%$ of the ocean surface. Palaeoceanographic data have revealed that this was not always the case. Before Drake Passage opened due to continental drift about $30 \mathrm{Myr}$ ago the climate of the ocean was considerably warmer. In the course of the establishment of the Southern Ocean in its present shape the difference between surface and bottom temperatures in equatorial regions changed from about $7^{\circ} \mathrm{C}$ to its present value of about $26^{\circ} \mathrm{C}$ (e.g. Berger 1981). The polar climate of the Southern Hemisphere became increasingly colder by the growth of glacial ice on the Antarctic continent and the gradual development of the sea ice cover around it, leading to the formation of deep cold water masses propagating as Antarctic Bottom Water (AABW) to the adjacent northern ocean basins. The opening of Drake Passage also established the strongest and longest current system in the world ocean, the Antarctic Circumpolar Current (ACC), with a volume transport of roughly $130-140 \mathrm{~Sv}\left(1 \mathrm{~Sv} \cong 10^{6} \mathrm{~m}^{3} \mathrm{~s}^{-1}\right)$, extending around the globe with a length of roughly $24000 \mathrm{~km}$. As the most important link between the ocean basins of the Atlantic, Pacific and Indian Oceans, the ACC serves as a conduit of all active and passive oceanic tracers which affect Earth's climate, notably heat and salt which strongly influence the oceanic mass stratification, circulation and consequently the ocean heat transport, and the greenhouse gas carbon dioxide. But in contrast to this strong zonal exchange

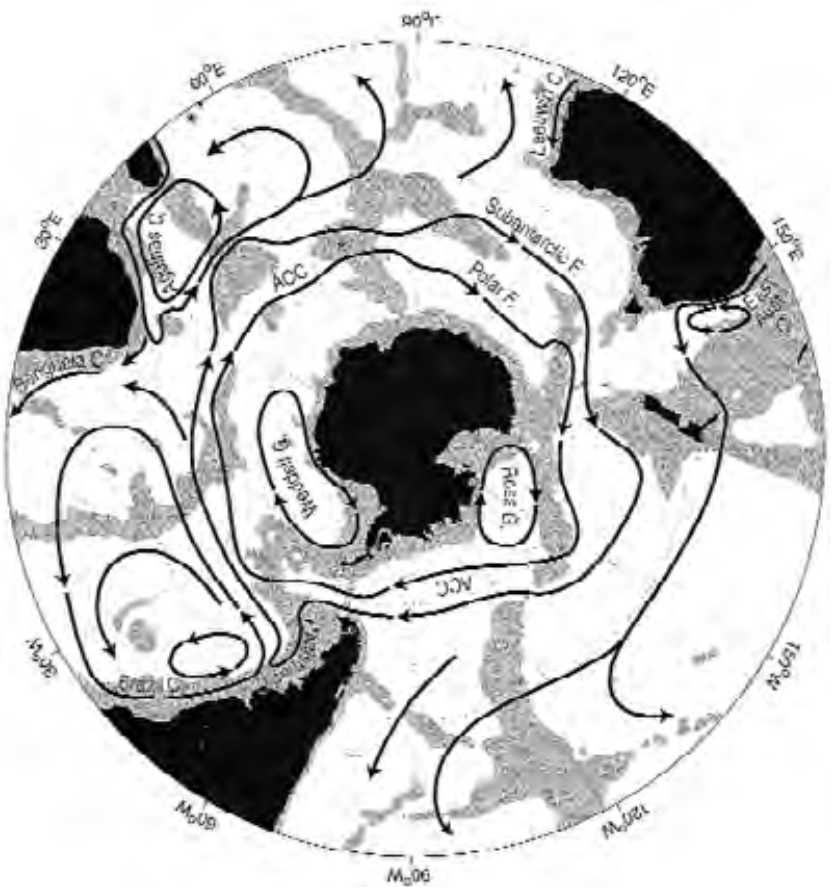

Fig. 1. Schematic map of major currents in the southern hemisphere oceans south of $20^{\circ} \mathrm{S}$. Depths shallower than $3500 \mathrm{~m}$ are shaded. The two major cores of the Antarctic Circumpolar Current (ACC) encircling Antarctica are shown: the sub-Antarctic Front and Polar Front. $\mathrm{F}=$ front, $\mathrm{C}=$ current and $\mathrm{G}=$ gyre. From Rintoul et al. (2001). 


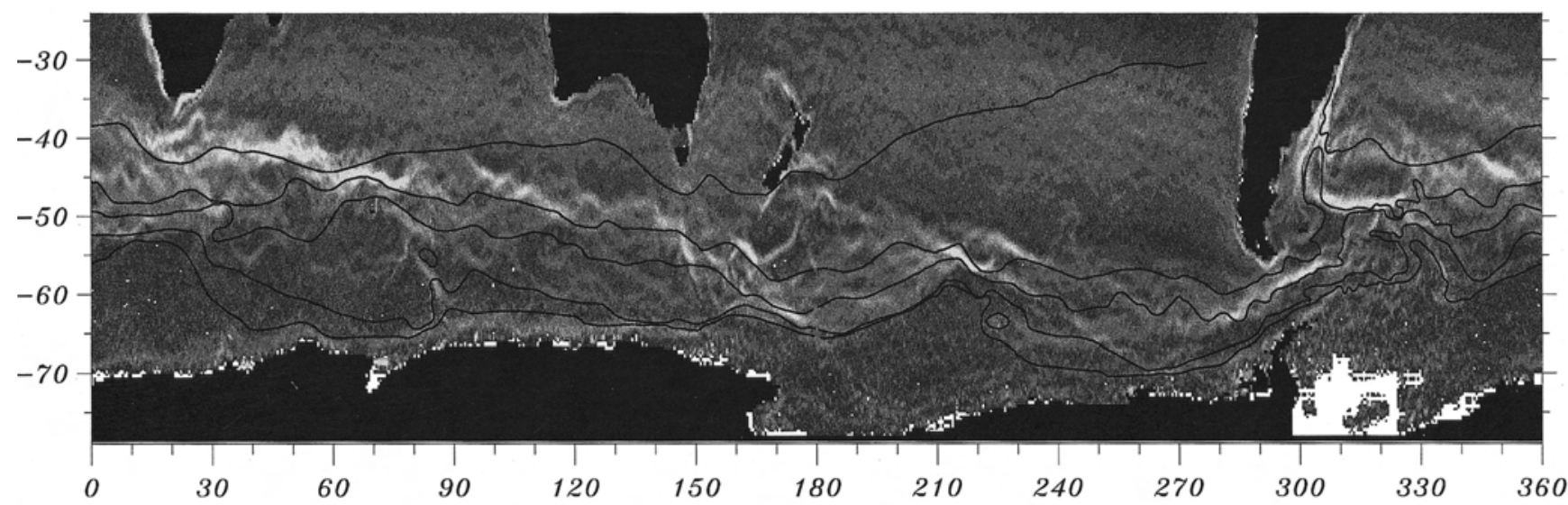

Scale: $\mathrm{mK} / \mathrm{km}$

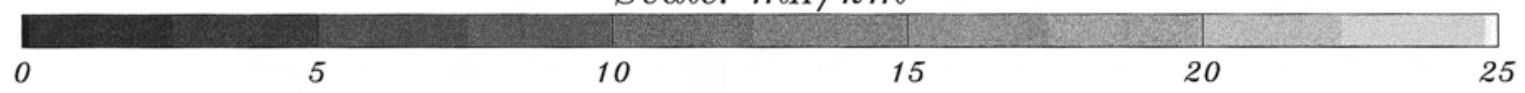

Fig. 2. Magnitude of the mean sea surface temperature gradient, from 44 months of observations by the Along-Track Scanning Radiometer on the ERS-1 satellite. Superposed are positions of (from north to south) the Subtropical Front, sub-Antarctic Front, Polar Front, South ACC Front, and southern boundary of the ACC, taken from Orsi et al. (1995). From Hughes \& Ash (2001).

brought about by the deep reaching and strong zonal current these same characteristics of the ACC act to limit meridional exchange and tend to isolate the ocean to the south from heat and substance sources in the rest of the world ocean.

The ACC system is sketched in Fig. 1 by its major fronts. These are traced by the regionally (and temporally, see Section 3) highly variable surface temperature gradient displayed in Fig. 2, which shows that the ACC is a fragmented system of more or less intense jet streams. The thermal fronts have a close correspondence in density and extend to depth, in most places to the bottom (see Fig. 3), but can also be correlated with surface elevations as detected in satellite altimetry data (e.g. Gille 1994). The ACC resides mainly in the two circumpolar fronts, the sub-

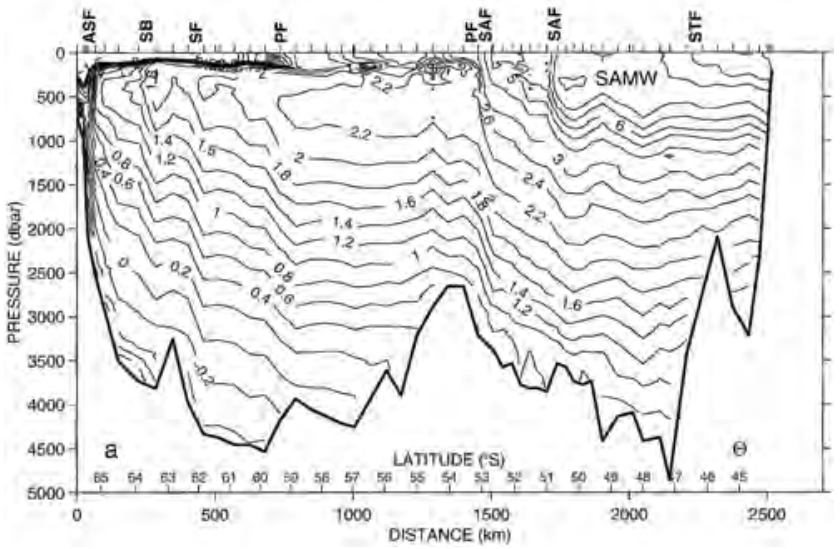

Antarctic Front and the Polar Front, which, due to regional and temporal variability, appear as multiple branches in the hydrographic section of Fig. 3.

From Fig. 3 it becomes apparent that watermass properties do penetrate across the ACC and, in fact, there is a prominent meridional circulation associated with the predominantly zonal ACC. It was described as early as 1933 by Sverdrup (see also Sverdrup et al. 1942) and has lately been interpreted as the Southern Ocean part of the 'global conveyor belt' circulation (Gordon 1986, Broecker 1991, Schmitz 1995). The 'sliced cake' view of the Southern Ocean watermasses and their propagation shown in Fig. 4 (Gordon 1999) presents this overturning circulation by the distribution of salinity and temperature of Antarctic Intermediate Water (AAIW), Circumpolar Deep Water



Fig. 3. Properties versus pressure along the WOCE SR 3 repeat section between Australia and Antarctica $\left(\approx 140^{\circ} \mathrm{E}\right)$. Left: potential temperature $\left({ }^{\circ} \mathrm{C}\right.$; contour interval is $1^{\circ}$ for $\Theta>3^{\circ} \mathrm{C}$, and $0.2^{\circ}$ for $\Theta<2.6^{\circ}$ ). Right: salinity (on the practical salinity scale, contour interval is 0.1 for solid contours, and 0.02 for dashed contours). From Rintoul et al. (2001). 


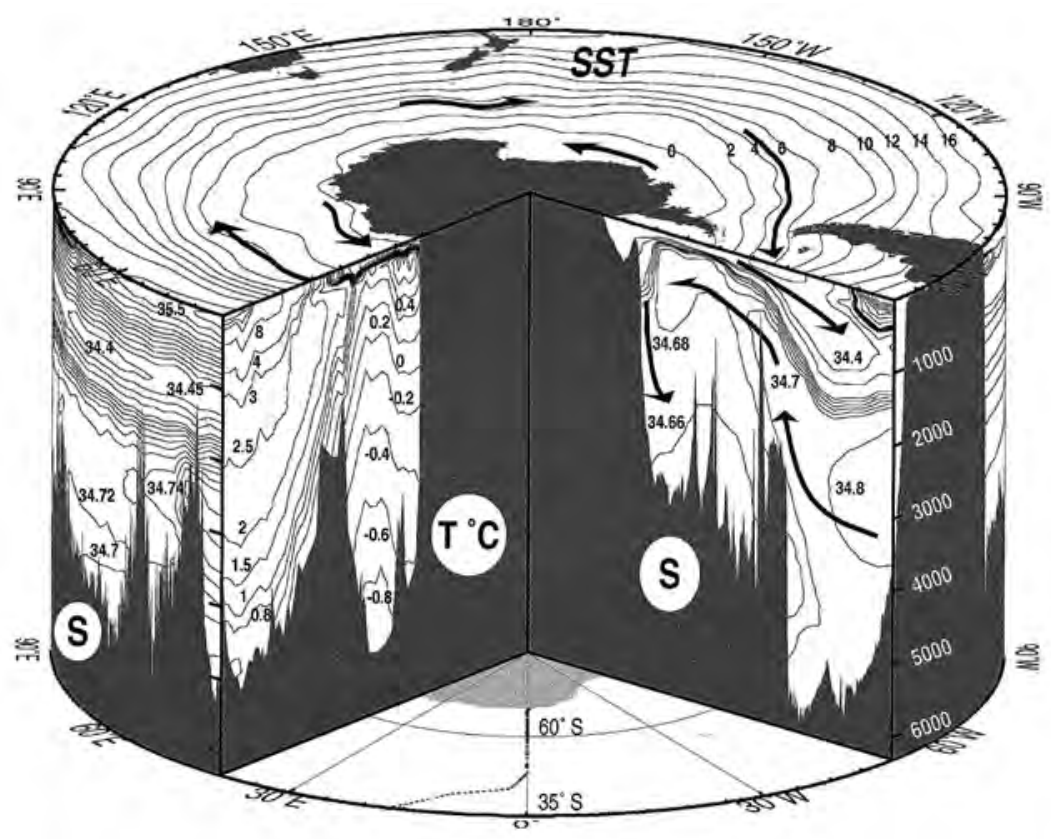

Fig. 4. A 'cake' with large slice removed view of the Southern Ocean. Isotherms of the annual average sea surface temperature $\left(\mathrm{SST}{ }^{\circ} \mathrm{C}\right)$ are shown on the plane of the sea surface. The core of the eastward flowing ACC and associated polar front occurs near the $4^{\circ} \mathrm{C}$ isotherm. The right plane of the slice shows salinity $(\mathrm{S})$. These data are derived from the oceanographic observations along the Greenwich Meridian shown on the floor of the figure (dots, from Fahrbach et al. 1994). Deep relatively saline water, > 34.7 (Circumpolar Deep Water CDW, arrow) spreads poleward and upwells towards the sea surface. It is balanced by a northward flow of lower salinity waters, $<34.4$ near $1000 \mathrm{~m}$ (Antarctic Intermediate Water AAIW, arrow) and by sinking of slightly lower salinity water along the continental slope of Antarctica (arrow). This process (salty water in, fresher water out) removes the slight excess of regional precipitation from the Southern Ocean. Along the left plane is temperature $\left(\mathrm{T}^{\circ} \mathrm{C}\right)$ based on data collected at the same points as used for the salinity section. Shallowing of the isotherms is evident as the deep water rises up towards the sea surface. There it is cooled and sinks flooding the bottom layers with waters of less than $0^{\circ} \mathrm{C}$. This cold bottom water spreads well into the global oceans (Antarctic Bottom Water AABW). Along the outer edge of the figure, latitude $35^{\circ} \mathrm{S}$, is salinity (S). The low salinity water (AAIW) is shown as the less than 34.45 band near $1000 \mathrm{~m}$. More saline deep water is seen spreading southward near the $4000 \mathrm{~m}$ depth. From Gordon (1999).


Fig. 5. Left: section integrated (south to north) baroclinic transport relative to the deepest common level for SR1 (Drake Passage) and SR3 (Australia to Antarctica) from various hydrographic section along SR1 and SR3 between 1991 and 2001. The location and names for the fronts are according to Orsi et al. (1995). Right: transport in neutral density classes. For SR3 the data of the various surveys are shown as bins, for SR1 only the mean transport profile of the cruises is given (full line). From Cunningham et al. (2003) and Rintoul \& Sokolov (2001). 
(CDW) and $\mathrm{AABW}$. The figure also depicts the circulation and nicely illustrates one of the central questions of Southern Ocean science: how do water properties (heat, salt, nutrients and other chemical substances) cross the strong deep reaching ACC? We will address this question of mass and property balances in Section 5, which is closely connected to the question of the dynamical balance of the ACC (treated in Section 3): what are the forces driving the zonal current, which act as brake and what physical mechanisms are responsible for the deep reaching current profile?

It should be borne in mind that the answer to these questions will not necessarily give insight into the problem of the relation of the magnitude of the zonal transport of water by the ACC to the flux of (zonal) momentum through the system (as the balanced transfer of money to and from a bank account does not determine the account balance). We discuss the dependence of transport on forcing functions and present a new simple linear transport model (Section 4).

In this paper we review some of the concepts and theories which are currently discussed for the circulation of the Antarctic Current system. It expands and complements other recent reviews of the ACC system (e.g. Olbers 1998, Rintoul et al. 2001) and summaries of the ACC dynamics contained in original research articles (e.g. Gnanadesikan \& Hallberg 2000, Tansley \& Marshall 2001). But we should point out that this review does not cover all the current research on the ACC, e.g. we do not address regional properties of the ACC system and its temporal variability; we not report on teleconnections and links of the Southern Ocean with the global ocean circulation and the possible dependence of the stratification and transport of the ACC on remote conditions and mechanisms.

\section{The zonal transport}

The meridional momentum balance of the ACC is basically geostrophic, i.e. the zonal current velocity (at each geopotential level) is related to the meridional pressure gradient, resulting from a dip of about $1.5 \mathrm{~m}$ (from north to south) of the sea surface across the current system, and the gradient of density in the fronts as, for example, can be inferred from temperature and salinity in the SR3 section in Fig. 3. The surface pressure gradient yields an overall eastward surface velocity and the mass stratification yields a positive shear $\left(u_{g}\right)_{z}=(g / f) \rho_{y}$ of the geostrophic part of the current $^{1}$, the velocity thus diminishes with depth but generally not as strongly as to imply a reversal of the flow. The above 'thermal wind relation' is utilized to infer from hydrographic section data the 'baroclinic' transport (normal to the section and referred to a common depth) or the DCL transport (referred to the bottom depth or deepest common

\footnotetext{
${ }^{1}$ We work with the Boussinesq approximation. Density, pressure and stresses are divided by a constant reference density.
}

level (DCL) for station pairs) of the ACC. Various attempts have been made to determine the absolute or net transport by taking reference velocities from moorings or LADCP (Lowered Acoustic Doppler Current Profiler) or by levelling bottom pressure gauges (see the recent discussion of Cunningham et al. 2003). Prior to WOCE most efforts were made in the International Southern Ocean Studies (ISOS) experiment at Drake Passage (Whitworth 1983, Whitworth \& Peterson 1985). More recently, transport estimations have been made at Drake Passage (WOCE SR1) and the section between Australia and Antarctica (WOCE SR3, see Fig. 3) at $140^{\circ} \mathrm{E}$, where multiple surveys have been made during WOCE.

The average DCL transport of SR1 for six hydrographic sections across Drake Passage (see Fig. 5) is $136.7 \pm 7.8 \mathrm{~Sv}$, with about equal contributions from the Polar Front (57.5 \pm $5.7 \mathrm{~Sv})$ and the sub-Antarctic Front $(53 \pm 10 \mathrm{~Sv})$ (Cunningham et al. 2003). The analysis of ISOS and WOCE data (spanning 25 years) gave no indication of significant trends or unsteadiness. Following Rintoul \& Sokolov (2001) the mean transport south of Australia at SR3 is $147 \pm$ $10 \mathrm{~Sv}$ (relative to a 'best guess' reference level: at the bottom except near the Antarctic margin, where a shallower level is used consistent with westward flow over the continental slope and rise). It is about $13 \mathrm{~Sv}$ larger than the ISOS estimate of absolute transport through Drake Passage and about $10 \mathrm{~Sv}$ larger than the SR1 DCL transport (see Fig. 5). The transport south of Australia must be larger than that at Drake Passage to balance the Indonesian throughflow, which is believed to be of order $10 \mathrm{~Sv}$. However, given the remaining uncertainty in the barotropic flow at both locations, the agreement is likely to be fortuitous. Variability of transport at SR3 has been detected in a six year record of repeat hydrographic section (Rintoul et al. 2002). It is fairly small (1-3 Sv). Figure 5 shows also the contribution of transport in the main classes of watermasses. In both sections the CDW range carries most of the zonal transport and no systematic temporal change of the relative contribution could be detected.

Monitoring the transport through Drake Passage is by now a standard diagnostic of numerical global ocean models. The resulting values are spread over a large range from well under $100 \mathrm{~Sv}$ to well over $200 \mathrm{~Sv}$. The reasons for this diversity are not fully understood. But wind forcing and thermohaline processes (Cai \& Baines 1996, Gent et al.

Table I. Volume transport of the ACC, diagnosed from some eddy permitting and eddy resolving ocean-only models.

\begin{tabular}{lccl}
\hline Model & Resolution & ACC transport & Reference \\
\hline FRAM & $1 / 4^{\circ} \times 1 / 2^{\circ}, 32$ levels & $195 \mathrm{~Sv}$ & FRAM Group 1991 \\
SC 92 & $1 / 2^{\circ}, 20$ levels & $180 \mathrm{~Sv}$ & Semtner \& Chervin 1992 \\
SC 92 & $1 / 4^{\circ}, 20$ levels & $163 \mathrm{~Sv}$ & Semtner \& Chervin 1992 \\
POP 11 & roughly 10 km in & $136 \mathrm{~Sv}$ & Maltrud et al. 1998 \\
& Drake Passage, 20 levels & & \\
OCCAM & $1 / 4^{\circ}, 36$ levels & $152 \mathrm{~Sv}$ & Fox et al. 2000 \\
\hline
\end{tabular}


2001), model resolution and the representation of topography (Best et al. 1999), as well as the parametrization of subgrid scale tracer fluxes (e.g. Danabasoglu \& McWilliams 1995, and Fritzsch et al. 2000) are known to be important factors. Most eddy resolving models (or 'permitting' since the achieved resolution does not resolve all of the relevant eddy scales) yield transport values closer to but mostly above the observations (see Table I).

Integrating the thermal wind balance $\left(u_{g}\right)_{z}=(g / f) \rho_{y}$ twice vertically we get

$$
U_{g}=\int_{-h}^{0} u_{g} d z=h u_{g}(z=-h)-\frac{g h}{f} \rho(z=-h) \frac{\partial h}{\partial y}-\frac{g}{f} \frac{\partial}{\partial y} \int_{-h}^{0} z \rho d z
$$

for the geostrophic transport (per unit length along the section). The geostrophic velocity at the bottom can be expressed by the gradient of pressure taken at the bottom, $u_{g}(z=-h)=-(p y)_{-h} / f$, which can be combined with the second term to yield

$$
U_{g}=-h \frac{\partial p_{b}}{\partial y}-\frac{\partial \chi}{\partial y}
$$

In this relation the geostrophic transport is expressed by the gradients of bottom pressure $p_{b}=p(z=-h)$ and the density moment

$$
\chi=g \int_{-h}^{0} z \rho d z \text { which is the total baroclinic potential }
$$
energy (referred to $z=0$ ). But $U_{g}$ is not the total transport, which is the integral of absolute velocity from top to bottom. The total volume transport through a section also contains the Ekman transports (due to wind stress and
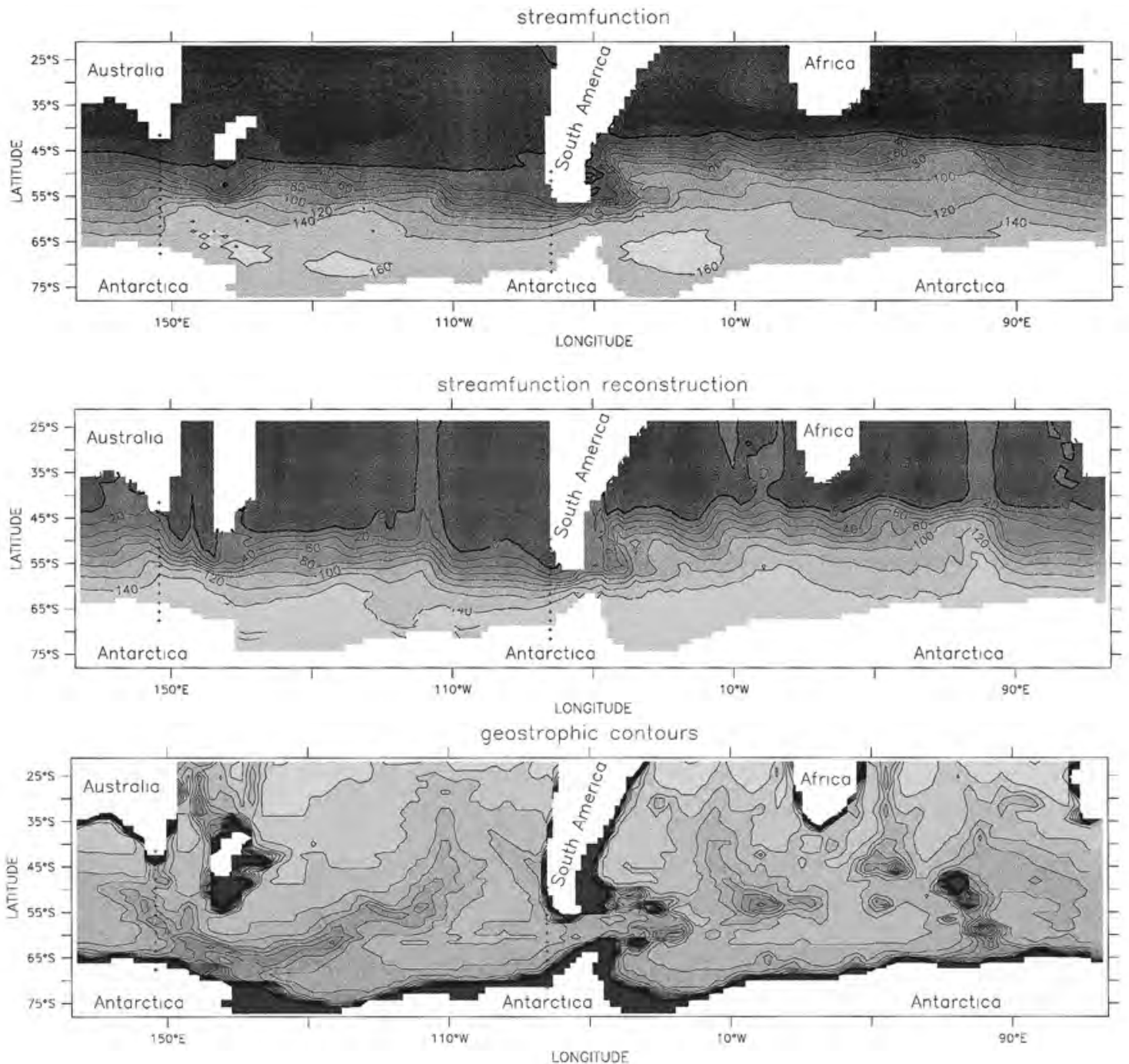

Fig. 6. Streamfunction $\psi$ [upper panel] and reconstruction of the streamfunction with (4) [middle panel]. The data are from a global OGCM with variable resolution $\left(1^{\circ} \times 2^{\circ}\right.$ at Drake Passage latitudes, 25 levels, MOM code). Geostrophic contours $f / h$ are shown in the lower panel. There are two prominent regions where geostrophic contours are blocked by continents: the region between Australia and Antarctica, and Drake Passage between South America and Antarctica. Here, the ACC must cross geostrophic contours. Although the geostrophic contours are not blocked there, the ACC also crosses the geostrophic contours at the East Pacific Rise and several other locations. From Borowski et al. (2002). 
frictional bottom stress) normal to the respective section and other contributions induced by nonlinearities and lateral friction. In large-scale currents the latter two are usually small and for north-south sections the Ekman contributions can be neglected (for predominantly zonal winds). But turning from the section coordinate $y$ to the general case we add to the geostrophic transport the Ekman parts and get the expression for the total transport $U$ in vector form,

$$
\begin{aligned}
f \boldsymbol{k} \times \boldsymbol{U} & =-f \nabla \psi=-h(\nabla p)_{-h}-g h \rho(z=-h) \nabla h-\nabla \chi+\tau_{0}-\tau_{b} \\
& =-h \nabla p_{b}-\nabla \chi+\tau_{0}-\tau_{b}
\end{aligned}
$$

The total transport conserves mass and is thus representable by a streamfunction $\psi$. With the approximation made so far (neglecting lateral stresses and nonlinearities) the above equation is the vertically integrated balance of momentum.

The relative importance of the different pressure gradient contributions in Eq. (1) or (2) to geostrophic transport has been addressed by Borowski et al. (2002). They argue on the basis of the balances of barotropic momentum and vorticity that the deep transport $h(\nabla p)_{-h}$ in Eq. (3) across geostrophic contours $f / h$ should be small if these are blocked by continents (as in Drake Passage and other places in the path of the ACC, see lowest panel of Fig. 6). Then, neglecting the variation of $f$ on the lhs of Eq. (3) and the deep and the Ekman transports on the rhs and integrating along a section of constant bathymetry $h=$ const, we find that the transport normal to such a contour is related to the difference of baroclinic potential energy between the ends, i.e. $f_{0} \Delta \psi \sim \Delta \chi$, a relation which we encounter again in Section 3.5. In models with simplified geometry such conditions can easily be established. In a series of experiments with zonal channel geometry (see Fig. 20), but also in global coarse resolution OGCMs, Borowski et al. (2002) could grossly verify the relation

$$
f \frac{\partial \psi}{\partial y}=\frac{\partial \chi}{\partial y}+g h \rho(z=-h) \frac{\partial h}{\partial y} \approx \frac{\partial \chi}{\partial y}
$$

between the meridional gradients of streamfunction and potential energy. Figure 6 compares the transport pattern (upper panel) and its reconstruction via Eq. (4) (middle panel) in a global coarse resolution OGCM. While there are clear differences in the closed basins of the major oceans, the overall agreement of the streamfunction and its reconstruction is rather good within the $\mathrm{ACC}$ region ( $\psi$ and its reconstructed values from the gradient of potential energy coincide within 10\%). By and large the contribution from the bottom pressure gradient to the transports is thus small.

\section{What is so special about the dynamics of the ACC?}

The zonal periodicity of the Southern Ocean, creating a circumpolar pathway of watermasses to circle the globe and allowing the ACC to play a major part in the conveyor belt circulation has already been mentioned. But the zonality also acts as a brake. In the basins which are zonally blocked by continents there is a meridional exchange of heat accomplished by the time mean gyre current systems. There is no such mean transport of heat across the latitudes of the ACC (DeSzoeke \& Levine 1981). Instead, the loss of heat from the ocean in the area south of the ACC must be carried across the current by smaller-scale and/or time varying features in the current field, usually summarized as the meso-scale eddy field. Transient eddies with scales tens to a few hundred kilometres (much larger than the baroclinic Rossby radius which is of order $10 \mathrm{~km}$ in the Southern Ocean, Houry et al. 1987) are a very prominent feature along the path of the ACC. There are also small stationary features, sometimes attached to outstanding topographic peculiarities, which scale in the category mesoscale eddies. With exception of the western boundary currents in the subtropical gyres the variance of transient features in the ACC dominates the global distribution of variability of surface displacement obtained from satellite altimetry, particular in areas of shallow rough topographic obstacles (e.g. Gille et al. 2000) and meridional excursions in the path of the flow. Estimates of the meridional eddy heat flux from a number of moored instruments confirmed the southward transfer with sufficient magnitude to close the overall heat budget (see Fig. 7). Recently Gille (2003) confirmed this
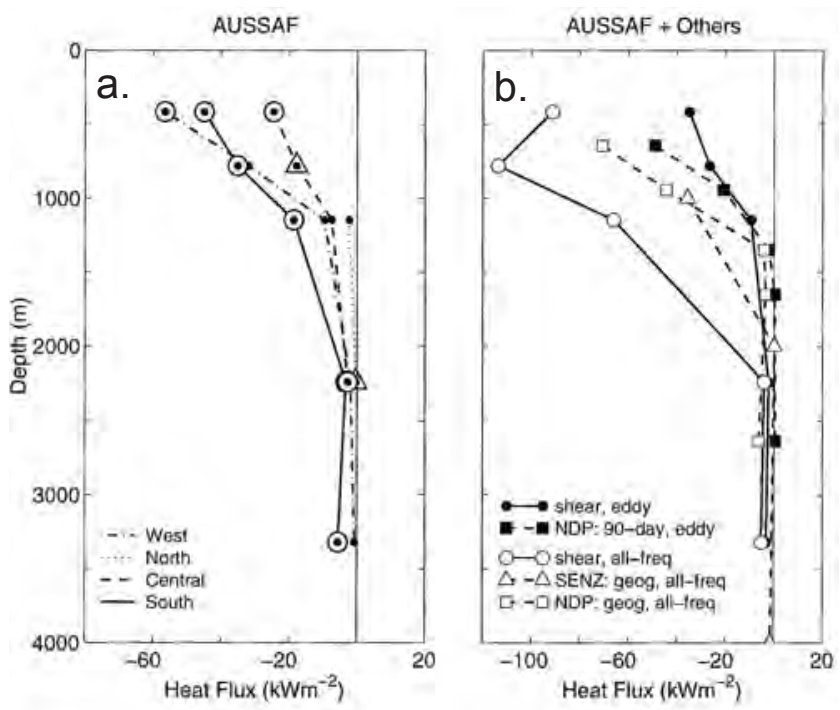

Fig. 7. Profiles of cross-stream eddy heat flux observed in the AUSSAF experiment. a. four AUSSAF moorings, symbols indicate statistical significance (triangle 95\%, circle 90\%), b. representative profiles from all available SAF sites: AUSSAF (circles), south-east of New Zealand (SENZ: Bryden \& Heath 1985, triangles), northern Drake Passage (NDP: Nowlin et al. 1985, squares). From Phillips \& Rintoul (2000). [geog: northward velocity is used, shear: velocity normal to mean current shear is used; the correlations are either determined from all frequencies or from a bandpass covering the eddy frequencies]. 




enormous extrapolation from point observations to the circumpolar area by determining the eddy heat flux from a combination of climatological hydrographic data and the ALACE (Autonomous LAgrangian Circulation Explorer) float trajectories tracing out the Southern Ocean in the last decade.

\subsection{Transient and standing eddies}

In the description of the global atmospheric circulation it is

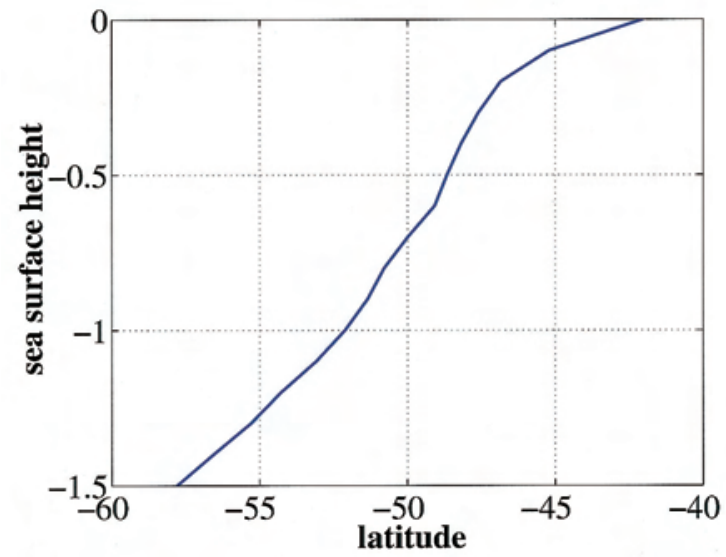

Fig. 8. The sea surface height in the Southern Ocean of a simulation with the POP ocean model. Contour interval is $0.1 \mathrm{~m}$. The contours between $-1.0 \mathrm{~m}$ and $0 \mathrm{~m}$ are as solid lines. The plot (above) shows the mean latitude associated with the SSH contours. These latitudes are used in Fig. 9 as lateral coordinate.

custom to reduce the information contained in observations by considering zonally averaged time mean fields and deviations from it (see e.g. Peixoto \& Oort 1992), e.g. the meridional velocity $v$ is split into its zonal-plus-time mean $\bar{v}$ and deviation $v^{*}$ so that $v=\bar{v}+v^{*}$. The mean meridional heat flux (divided by $\rho c_{p}$ ) is then $\overline{v T}=\bar{v} \bar{T}+\overline{v^{*} T^{*}}$ which identifies a flux achieved by the mean fields and a flux induced carried by the covariance of the deviation fields (the 'eddies'). Clearly, the combined zonal-plus-time mean of $v^{*}$ vanishes, i.e. $\widehat{v^{*}}=0$, but the
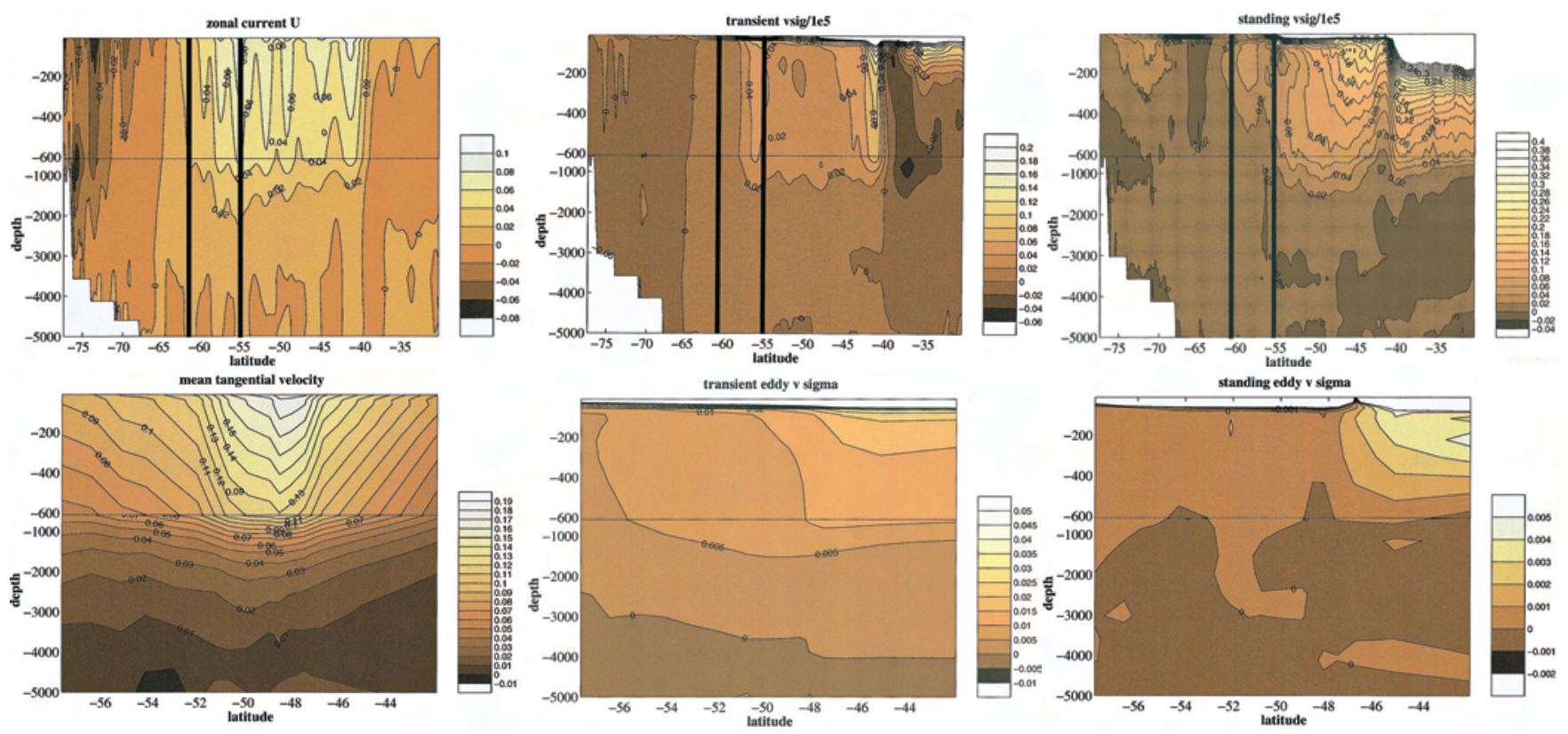

Fig. 9. Comparison of zonally averaged fields [upper panels] and mean fields constructed from an ACC path following averaging procedure [lower panels]. The left panel displays the mean current, the middle and right panels display the transient and standing components of the potential density flux (note that contour intervals are different). The heavy lines in the upper panels mark the latitudes of Drake Passage. The path following average fields is displayed with respect to the mean latitudes of the corresponding SSH contours, as given in the plot in Fig. 8. Units: velocity in $\mathrm{ms}^{-1}$, density fluxes in $\mathrm{m}^{2} \mathrm{~s}^{-1}$. 
time mean alone does not vanish. Denoting the temporal average by cornered brackets we get a separation into a time mean ('standing eddy') component and the time varying ('transient eddy') component, $v^{*}=\left\langle v^{*}\right\rangle+v^{\prime}$ ', and likewise for the 'eddy' heat flux, $\overline{v^{*} T^{*}}=\overline{\left\langle v^{*}\right\rangle\left\langle T^{*}\right\rangle}+\left\langle v^{\prime} T^{\prime}\right\rangle$ with the contributions from standing and transient eddies.

Motivated by the zonal unboundedness of the ACC this separation of fields and covariances has been applied to data (from models since synoptic maps of ACC properties do not exist) for the belt of latitudes passing Drake Passage (e.g. Killworth \& Nanneh 1994, Stevens \& Ivchenko 1997, Olbers \& Ivchenko 2001). We elucidate the typical results of zonal averaging using results from the global ocean POP model (Parallel Ocean Program, see Maltrud et al. 1998) which marginally resolves the transient eddy field with a resolution of roughly $6.5 \mathrm{~km}$ in polar latitudes. The time mean sea surface topography of the simulation is shown in Fig. 8, revealing a quite realistic ACC (compare to Fig. 2) as a collection of strong, regionally bounded jets which break up at topographic features and in summary pass Drake Passage but do not at all follow the corresponding belt of latitudes. Consequently, in the zonally averaged picture (Fig. 9 upper panels) many details of the ACC current system are lost. The averaged state in the Drake Passage band of latitudes is picked from the stronger features at the southern rim of the ACC in that latitude interval and thus misses most of its circumpolar structure. Moreover, the average does not at all represent the local structure of the current in Drake Passage. The transport of the ACC through Drake Passage is $130 \mathrm{~Sv}$ in this POP simulation, which is very close to observations. In contrast, the transport of the zonally averaged current is only about $50 \mathrm{~Sv}$.

Most of the ACC actually finds its representation in the averaged picture at latitudes north of the Drake Passage belt (see for example the zonally averaged zonal current in Fig. 9). As a consequence we have standing eddy contributions which are strong compared to the transient eddy contributions. This is exemplified in Fig. 9 by the eddy density flux $\overline{\left\langle v^{*}\right\rangle\left\langle\rho^{*}\right\rangle}$ and $\left\langle v^{\prime} \rho^{\prime}\right\rangle$, respectively. In the Drake Passage belt these fluxes are of comparable size and northward - at blocked latitudes - the flux of the standing eddies overwhelms the flux of the transient eddies by an order of magnitude.

The lower suite of panels in Fig. 9 displays the same fields using an alternative average which is oriented along the contours of the time mean sea surface height ( $\mathrm{SSH}$; the POP code has a free surface implemented): we show the mean tangential velocity and the component of the density flux by standing and transient eddies which is normal to the SSH contours ('standing' now refers to the deviations from the convoluted path). This path following average clearly captures more of the properties in the ACC region than a zonal average. A similar streamwise average analysis is presented in Ivchenko et al. (1998) and Best et al. (1999). The mean tangential velocity collects all jets into a strong current - surprisingly with one single core. It is eastward everywhere and centred at the height contour $-0.5 \mathrm{~m}$ (mean latitude of $-49^{\circ}$ ) with the highest speeds at the surface of about $0.2 \mathrm{~m} \mathrm{~s}^{-1}$ which is two times the maximum of the zonally averaged zonal velocity. The eddy fluxes, shown in the middle and left panels, demonstrate that time mean and transient field are separated to a large extent: the standing eddy component is still non-zero (because the flow slightly turns with depth) but is clearly much diminished compared with the zonal mean and negligible compared to the transient component of the path following mean.

In summary, we conclude that the zonal average does not separate the time mean and the transient motion in a simple way. Zonal mean, standing eddy and transient eddy components arise and the standing eddy component is a major player. Dynamically it belongs to the time mean flow but it overrides the transient component. When analyzing dynamical balances in the latitudinal-longitudinal coordinate system standing and transient components have their physical meaning (e.g. in the balance of zonal momentum which will be discussed in many places in this paper). But building models of a mean circulation in a zonally average framework is inherently hampered by an inadequate treatment of the standing eddy component. Because it is intractable to parameterizations it is generally neglected but is larger than the transient component for which reasonable parameterizations are known (Johnson \& Bryden 1989).

The path following (or convoluted) average produces a much clearer separation of the flow into time mean and transient components. In this framework the coordinate system is attached to the specific flow (the model $\mathrm{SSH}$ contours in the above example). Analysing balances or setting up models in the convoluted average frame is conceptually simpler because standing eddies can be neglected but the fields (velocities, fluxes etc) are oriented at the convoluted coordinates. For instance, in a convoluted average analysis we would consider the balance of the along-stream component of momentum with the alongstream component of wind stress entering, rather than the balance of zonal momentum. In the course of the paper we will frequently have recourse to these different averaging concepts.

\subsection{Interfacial and bottom form stress}

Eddies not only carry heat in the mean but also establish a transfer of momentum as well. While lateral eddy momentum fluxes turned out to be rather small (compared to the wind-stress) and indifferent in sign (Morrow et al. 1994, Phillips \& Rintoul 2000, Hughes \& Ash 2001) the ACC is the outstanding example in the ocean for diapycnal transport of momentum by eddies. Since the momentum imparted to the ocean surface layer by the strong zonal winds in the Southern Ocean cannot be balanced in the 


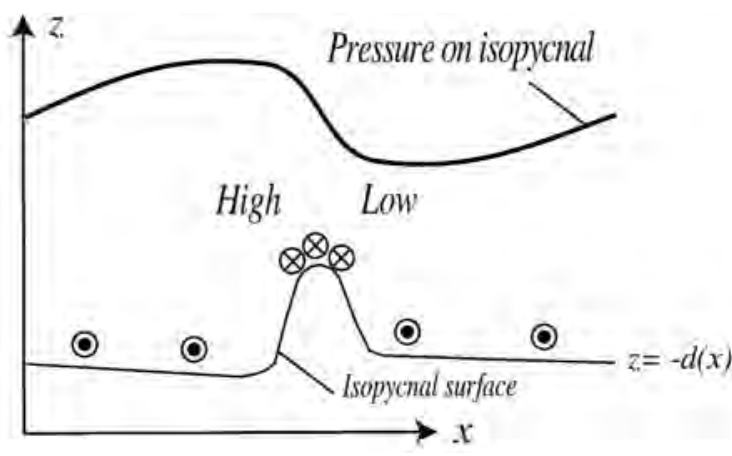

Fig. 10. Schematic demonstrating the interfacial form stress for an isopycnal interface in the water (shown is the zonal depth). There is higher pressure at the depth of the density surface where it is rising to the east compared with where it is deepening to the east. This results in an eastward pressure force (interfacial form stress) on the water below. This is related to the fact that the northward flow occurs where the vertical thickness of water above the density surface is small, and southward flow where the thickness is large, so there is a net southward mass flux at lighter densities due to the geostrophic flow. The same kind of pressure force acting on the sloping bottom topography leads to the bottom form stress. Redrawn from Rintoul et al. (2001).

Drake Passage belt by large scale zonal pressure gradients a consequence of the lack of zonal boundaries - and because lateral Reynolds stresses are too small for significant transport away from the ACC towards boundaries, a downward transfer of momentum is the only mechanism to prevent indefinite acceleration of the zonal flow in the surface layer. The diapycnal momentum transport cannot be achieved by small-scale three-dimensional turbulence (it would require viscosities far too large); it must be done by the eddies. Depending on the framework - layer or level coordinates - different kinds of eddy terms arise in the dynamical balances. We first present the dynamical balances for a layer framework, and later describe the corresponding physics in level coordinates (at the end of Section 3.3 and in Section 4.1).

The most important mechanism of momentum transfer in layer coordinates is the eddy interfacial form stress (IFS). It operates everywhere in the ocean where eddies are present and deform isopycnals but the unique sign and magnitude in the vast circumpolar area is truly outstanding. IFS transfers horizontal momentum across inclined (by eddies) isopycnals by fluctuations (by eddies) in the zonal pressure gradient. Imagine two interfaces (isopycnals) $z=-d_{I}(x)$ and $z=-d_{2}(x)$ along any circumpolar path with coordinate $x$ along it and integrate the (negative) pressure gradient $-p_{x}$ between the interfaces and around the path,

$$
\begin{aligned}
-\oint \int_{-d_{2}(x)}^{-d_{1}(x)} \frac{\partial p}{\partial x} d z d x & =-\oint\left[p\left(x, z=-d_{1}(x)\right) \frac{\partial d_{1}}{\partial x}-p\left(x, z=-d_{2}(x)\right) \frac{\partial d_{2}}{\partial x}\right] d x \\
& =\oint\left[d_{1} \frac{\partial}{\partial x} p\left(x, z=-d_{1}(x)\right)-d_{2} \frac{\partial}{\partial x} p\left(x, z=-d_{2}(x)\right)\right] d x
\end{aligned}
$$

to get its contribution to the rate of change of $x$-momentum in the corresponding volume. The pressure is taken at the isopycnal depth and its gradient appearing in the second formulation thus acts across the inclined isopycnal. Obviously, to get a non-zero $\overline{d p_{x}}$ (the overbar indicates the path and time mean) the pressure must vary at the isopycnal depth in a way that an out-of-phase part with respect to the depth variations is present (see Fig. 10). Evidently, the strip of ocean gains $x$-momentum by the amount $\overline{d_{1} p_{1 x}}$ from the fluid above $z=-d_{1}(x)$ and loses $\overline{d_{2} p_{2 x}}$ to the fluid below $z=-d_{2}(x)$. Thus, for infinitesimally distant isopycnals the vertical divergence of the interfacial form stress IFS $=\overline{p d_{x}}=-\overline{d p_{x}}$ enters the momentum balance. The mean depth $\bar{d}$ is not relevant, only the eddy component $d^{*}=d-\bar{d}$ contributes (also for pressure) so that IFS $=-\overline{d^{*} p^{*}{ }_{x}}$. The starred quantities contain the signal from the time-mean 'standing' eddies and the signal from the time varying transient eddies and the IFS may be separated accordingly.

Equating the zonal pressure gradient with the northward geostrophic velocity, $f v_{g}^{*}=p_{x}^{*}$, and the layer depth fluctuation with (potential) temperature anomaly, $d^{*}=\theta^{*} / \bar{\theta}_{z}$, we find that the IFS relates to the meridional eddy heat flux, $-\overline{d^{*} p^{*}{ }_{x}}=\left(f / \bar{\theta}_{z}\right) v_{g}^{*} \theta^{*}$. A poleward eddy flux of heat is just a downward transport of zonal momentum by IFS in the water column. These processes are strictly coupled. The transient eddies which carry the poleward heat flux shown in Fig. 7 thus establish a downward transport of momentum.

In summary, though horizontal pressure gradients can only establish a transfer of horizontal momentum in horizontal direction they do transport horizontal momentum across tilted surfaces from one piece of ocean to another. A layer bounded by tilted isopycnals is thus forced by stresses (IFS) at the bounding top and bottom surfaces (in the same way as the Ekman layer is driven by frictional stresses at top and bottom).

Deriving the relation Eq. (5) it was assumed that the isopycnal strip does not run into the bottom nor touches the sea surface. If this situation occurs additional pressure terms arise from the bounding outcrops. These terms present a flux of horizontal momentum through these boundaries into the strip. For the bottom contact the corresponding flux is part of the bottom form stress (see below).

Notice that the same mathematical operations used to derive Eq. (5) apply if the interface is solid as, for example, the ocean bottom at $z=-h(x)$. The bottom form stress $B F S=$ $-\overline{h p_{b x}}=\overline{h_{x} p_{b}}$ operates here to transfer zonal momentum out of the fluid to the solid earth (since $h$ is constant in time only the time mean bottom pressure is relevant). BFS works everywhere in the ocean where the submarine ocean bed is inclined but to be of significance the gradient of the bottom pressure (the normal geostrophic velocity) must be correlated to the ocean depth variations, or vice versa: the bottom pressure must be out of phase with the depth along the respective circumpolar path, e.g. there must be high 


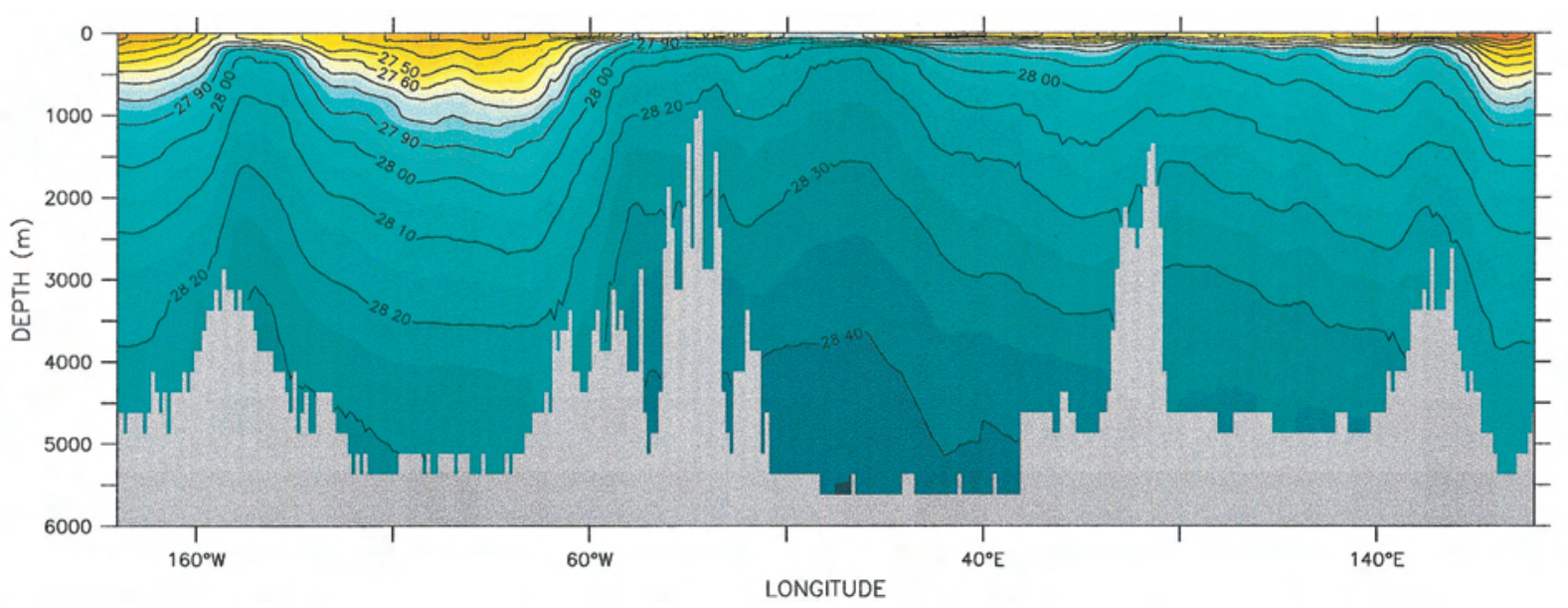

Fig. 11. Zonal section of the observed potential density at $60^{\circ} \mathrm{S}$, taken from the gridded data of the WOCE Hydrographic Special Analysis Center (SAC, see http://www.dkrz.de/ u241046/SACserver/SACHome.htni). The section is viewed from the south.

pressure at rising topography and low pressure at the opposite falling slope to the east to let eastward momentum leak out to the earth. A depth-pressure correlation can in fact be seen in circumpolar hydrographic sections passing through Drake Passage around Antarctica, as shown in Fig. 11. From the density $\rho$ we can infer the baroclinic bottom pressure $p^{c l i n}=g \int_{z}^{0} \rho d z$

contained in the mass stratification.

It is obvious in the section that there is more lighter water to the west of the submarine ridges than to the east. Surprisingly the $B F S$ derived from such a pattern accelerates the eastward current, acting thus in cooperation with the eastward wind stress - a feature of the ACC dynamics which will be reconsidered in the course of this paper.

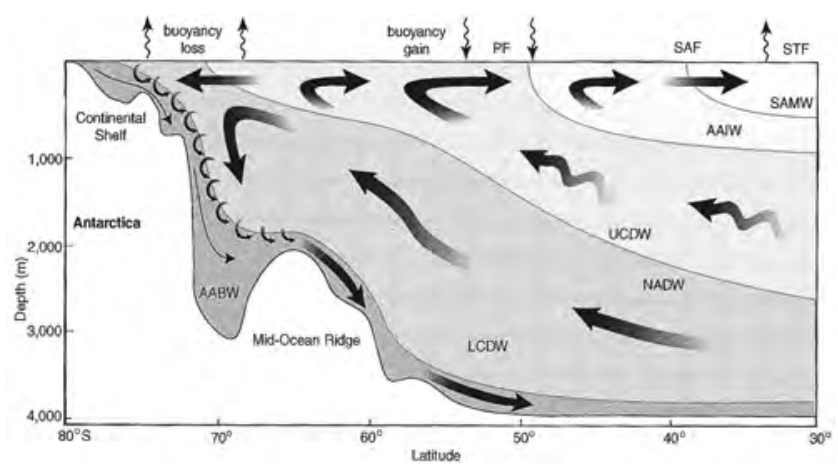

Fig. 12. A schematic view of the meridional overturning circulation in the layers of the Southern Ocean watermasses as an enlarged view of the cut in the 'cake' of Fig. 4. An upper cell formed primarily by northward Ekman transport beneath the strong westerly winds and southward transport in the Upper Circumpolar Deep Water (UCDW) layer is assumed 'unblocked' by topography in the three-layer model of Section 3.3. The lower cell driven primarily by formation of dense AABW near the Antarctic continent and inflowing NADW is assumed 'blocked' beneath the depth of the highest topography. From Speer et al. (2000).

\subsection{The dynamical balance of the zonal flow}

The IFS and BFS contributions to the physics of zonal currents can be elucidated by a simple conceptual model. Consider a strip of ocean from Antarctica to the northern rim of the ACC and split the water column into three layers (which may be stratified), separated by interfaces which ideally are isopycnals (see Figs $11 \& 12$ ). The upper layer from the sea surface $z=\eta_{0}=\zeta$ to some isopycnal at depth $z=-\eta_{1}$ and includes the Ekman layer, the intermediate layer with base at $z=-\eta_{2}$ lies above the highest topography in the Drake Passage belt (the range of latitudes which run through Drake Passage), and the lower layer reaches from $z=-\eta_{2}$ to the ocean bottom at $z=-\eta_{3}=-h$. We apply a time and zonal average to the balance equations of zonal momentum in the three layers and use Eq. (5) to get

$$
\begin{array}{r}
\quad-f \bar{V}_{1}=-\overline{\eta_{1}^{*} p_{1 x}^{*}}+\tau_{0}-\tau_{1}-R_{1} \\
-f \bar{V}_{2}=\overline{\eta_{1}^{*} p_{1 x}^{*}}-\overline{\eta_{2}^{*} p_{2 x}^{*}}+\tau_{1}-\tau_{2}-R_{2} \\
-f \bar{V}_{3}=\overline{\eta_{2}^{*} p_{2 x}^{*}}-\overline{h p_{b x}^{*}}+\tau_{2}-\tau_{b}-R_{3}
\end{array}
$$

where the depth and zonally integrated northward volume flux in each layer are denoted by $\bar{V}_{i}, i=1,2,3$. Furthermore, $p_{i}$ is the pressure at the respective layer depths, $p_{3}=p_{b}$ the bottom pressure and the overbar denotes time and ACC path following mean. Note that the surface term $\overline{\zeta p_{0 x}}$ drops out in the first equation because the surface pressure is $p_{0}=g \zeta$. As before, the star denotes the deviation from this average, $\tau_{0}$ is the wind stress, $\tau_{i}$ the frictional stresses at interfaces, $\tau_{3}=\tau_{b}$ the frictional bottom stress, and $R_{i}=\partial \overline{u_{i}^{*} v_{i}^{*}} / \partial y$ the divergence of the appropriate lateral Reynolds stress. We will assume that the interfacial friction stresses $\tau_{l}, \tau_{2}$ and the $R_{i}$ can be neglected (which is confirmed by measurements, e.g. Phillips \& Rintoul 2000, and eddy resolving models). The meridional circulation is defined by transports between isopycnals and is thus of Lagrangian quality. The wind-driven component $-\tau_{0} / f$ (the Ekman transport) in the top layer, a similar 
frictional transport $\tau_{b} / f$ in the bottom layer, and a geostrophic component in the bottom layer, associated with the bottom form stress $-\overline{h p_{b x}}$, also appear if the flow is averaged between geopotential (constant depth) levels (see end of this section). These Eulerian quantities form the Deacon cell of the Southern Ocean meridional overturning (see Döös \& Webb 1994 and Fig. 24).

Since $\Sigma_{i} \bar{V}_{i}=0$ by mass balance of the ocean part to the south (neglecting the very small effect of precipitation and evaporation on mass balance) the overall balance of zonal momentum is between the applied wind stress, the bottom form stress and the frictional stress on the bottom,

$$
\tau_{0}-\overline{h p_{b x}}-\tau_{b} \simeq 0
$$

The frictional stress $\tau_{b}$ and the here neglected Reynolds stresses are generally small in the ACC. Munk \& Palmen (1951) were the first to discuss this balance of momentum for the ACC (but surprisingly, much of the research on the ACC after Munk \& Palmen's article had forgotten the importance of the bottom stress and tried frictional balances, e.g. Hidaka \& Tsuchiya (1953), Gill (1968)). Hence, the momentum put into the ACC by wind stress is transferred to the solid earth by bottom form stress. The transfer is at the same latitude because the divergence of the Reynolds stress is small. This balance has been confirmed in most numerical models which include submarine topographic barriers in the zonal flow and have a realistic (small) magnitude of the Reynolds stress divergence. If the ocean bottom is flat (in models the bottom can be made flat) either bottom friction may get importance and/or the neglected Reynolds terms could come into play. Eddy effects seem to be unimportant in the vertically integrated

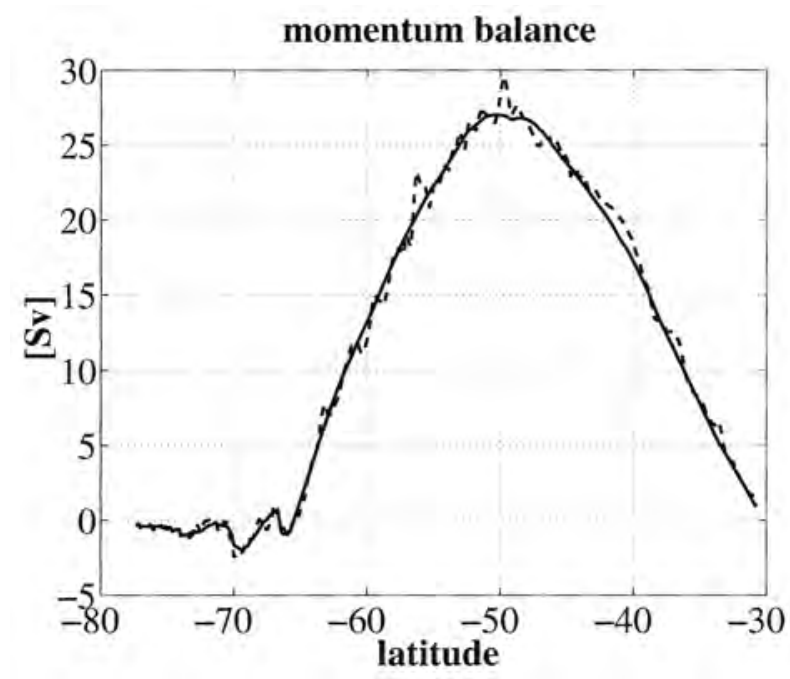

balance but it is worth mentioning that most coarse OGCMs do not confirm Eq. (7), see Cai \& Baines (1996). The reason is that such models use very large lateral viscosities so that the parameterized Reynolds stresses become large, even though the simulated current is broad and smooth.

Figure 13 exemplifies the total zonal momentum balance with results from the eddy resolving POP model (Parallel Ocean Program, Maltrud et al. 1998). It is instructive to write the pressure $p$ as sum of the baroclinic (density related) part $p^{c l i n}=g \int_{z}^{0} \rho d z$

and the barotropic (surface related) part $g \zeta$. While the total bottom form stress clearly takes out the momentum put in the ocean by wind stress we have seen above in Fig. 11 that the baroclinic part $\overline{h\left(p_{b}^{\text {clin }}\right)_{x}}$ does not have the corresponding sign: according to the phase shift of density with respect to the submarine topography the baroclinic bottom form stress should accelerate the eastward current. Indeed, this has been found in the analysis of the eddy permitting model FRAM (Fine Resolution Antarctic Model, Fram Group 1991). The right hand panel of Fig. 13 displays the balance with the pressure terms $\overline{h\left(p_{b}^{c l i n}\right)_{x}}$ and $g \overline{h \zeta_{x}}$ separated. Individually they are much larger than the zonal wind stress, by about an order of magnitude, but of opposite sign and thus they nearly cancel. This feature in the dynamical balance of the ACC will be further discussed in Section 4.2. A summary of the balance of zonal momentum in the ACC is displayed in Fig. 14.

A global perspective of the zonal balance is presented in the experiments which Bryan (1997) has performed with a non-eddy-resolving OGCM. In his findings the balance between zonal wind stress and bottom form stress prevails everywhere in the world ocean and likewise, we find in all

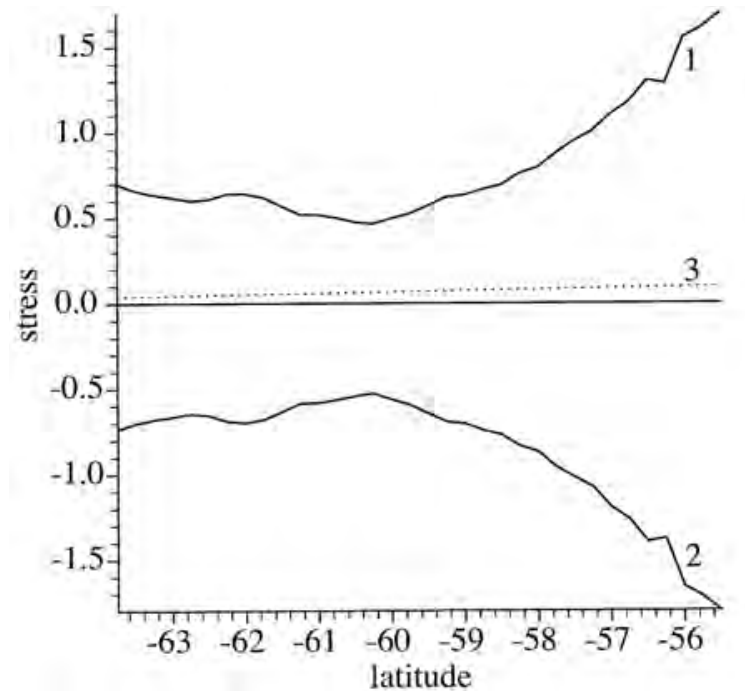

Fig. 13. Left: Vertically integrated balance of total momentum from the POP model in the Southern Ocean, indicating a balance between wind-stress [solid line] and negative bottom form stress [dashed] at each latitude (small deviations are due to Reynolds stress effects; the terms are normalized by $f_{0}=1.25 \times 10^{-4} \mathrm{~s}^{-1}$, units are Sv). From Olbers (2005). Right: vertically integrated balance of total momentum from the FRAM model in the Drake Passage belt: 1 is baroclinic form stress, 2 barotropic form stress, 3 zonal wind-stress (units Nm ${ }^{-2}$ ). From Stevens \& Ivchenko (1997). 


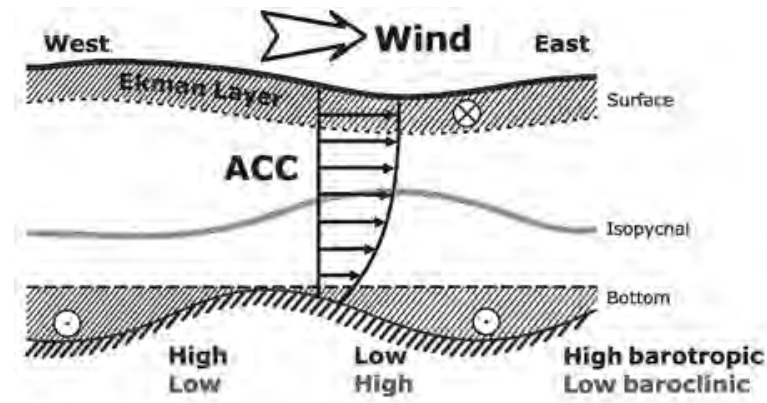

Fig. 14. Sketch of the zonal balance of momentum for the ACC: the flow establishes a high of barotropic (surface) pressure and a low of baroclinic (hydrostatic) pressure upstream of a zonal ridge and a corresponding low/high downstream. The associated barotropic and baroclinic bottom form stresses almost balance, their residual counteracts the wind stress. The wind drives a northward Ekman transport in the surface layer $(\otimes)$, and corresponding to the bottom form stress there is southward geostrophic return flow in the valleys between the ridges which partly block the zonal path $(\odot)$. The system is viewed from Antarctica.

experiments (run with different wind climatologies) the approximate cancellation of the barotropic and baroclinic form stresses which individually are an order of magnitude larger than the wind stress (see fig. 11 of Bryan 1997). It is noteworthy that the signature of the momentum balance found here for the ACC - with driving by the baroclinic form stress and braking by the barotropic - is only found in Bryan's results poleward of the subtropical gyres.

If, in addition to the assumptions of small $R_{i}$ and $\tau_{1}, \tau_{2}$ and $\tau_{b}$, the flow conserves potential density then there cannot be transport across isopycnals and the meridional transport in each layer must vanish, $\bar{V}_{i}=0$, by mass conservation. We find that the interfacial form stress $\overline{\eta_{i}^{*} p_{i x}^{*}}$ is vertically constant and equal to the wind stress $\tau_{0}$ and to the bottom form stress,

$$
\overline{\eta_{i}^{*} p_{i x}^{*}} \simeq \tau_{0} \simeq \overline{h p_{b x}}
$$

Then, in each layer, the meridional mass fluxes induced by wind stress and pressure gradients are compensated (in models with a flat bottom, the bottom form stress must be replaced by the frictional bottom stress in the above relation). This scenario of 'constant vertical momentum flux' is realized in quasigeostrophic layer models (Wolff et al. 1991, Marshall et al. 1993, Olbers et al. 2000, Olbers 2005 ) which are by construction adiabatic.

The real ocean is diabatic, i.e. there is mixing across isopycnals by small-scale turbulence and air-sea fluxes, but it is still in debate if it occurs predominantly between the outcropping isopycnals in the surface layer or in the interior as well (see Section 5). The meridional overturning transports $\bar{V}_{i}$ at a certain latitude circle can be non-zero only if there is exchange of mass between the layers south of the respective latitude - implying conversion of watermasses south of the ACC. In fact, by mass conservation, the $\bar{V}_{i}$ equal the net exchange with the neighbouring layers over the area south of the respective latitude. At the same time, the overturning transports imply a Coriolis force in the individual isopycnal layers which is in balance with the vertical divergence of the interfacial form stress. The divergence of the heat flux due to transient eddies can clearly be deduced from Fig. 7 (we have shown that roughly $-\overline{\eta^{\prime} p_{x}^{\prime}} \approx-\overline{f v_{g}^{\prime} \theta^{\prime}} / \bar{\theta}_{z}$ ). Eddy effects at the respective latitude and diabatic interior effects of smallscale turbulence occurring to the south must thus adjust according to mass and momentum requirements of the zonal current and the meridional overturning. The isopycnal analysis of the zonal momentum balance in the FRAM model by Killworth \& Nanneh (1994) can be taken to exemplify the importance of diabatic processes and the inapplicability of the 'constant vertical momentum flux' scenario: there is a net meridional circulation at all depths in balance with a divergent $I F S$ and wind stress (the latter influences also deeper isopycnal layers which outcrop at some longitude along the circumpolar path).

We have so far discussed the momentum balance in a Lagrangian framework by using isopycnal layers. But many dynamical concepts and the numerical models are written in an Eulerian framework where geopotential (depth) horizons are the vertical coordinates. Interfacial form stress is then invisible, and apart from Reynolds stress terms the zonally averaged balance of zonal momentum

$$
-f\{v\}=\frac{\partial\{\tau\}}{\partial z}-\frac{\partial}{\partial y}\left\{u * v^{*}\right\}-\frac{\partial}{\partial z}\left\{u * w^{*}\right\}+\sum_{\text {ridges }} \delta \bar{p}_{b}
$$

has no obvious signature of eddy effects at all. Here, $\tau$ is stress (vertical transport of zonal momentum by turbulent motions) in the interior. The sum of the bottom pressure differences $\delta \bar{p}_{b}$ is extended over all submarine ridges interrupting the integration path at depth $z$ (continents are included). Each ridge or continent contributes to the difference between the values on the eastern side and the western side, i.e. $\delta p_{b}=p\left(x_{E}, y, z=-h\right)-p\left(x_{W}, y, z=-h\right)$. The curly bracket operator denotes zonal integration on level surfaces and $a^{*}=a-\{a\} / L$ is the deviation (again standing plus transient eddy component), $L$ is the path length and the overbar is now only the time mean. From mass balance it may be shown that the vector $\{v\}=-\partial \phi / \partial z,\{w\}=\partial \phi / \partial y$ has a streamfunction $\phi$ (the meridional Eulerian streamfunction) despite possible interruptions of the zonal path by submarine topography. Equation (9) may then be integrated vertically from the surface to some depth $z$ to yield the balance for the depth interval from the surface to the level $z$,

$$
-f \phi=\left\{\tau_{o}\right\}-\{\tau\}-R+F
$$

where $R$ collects the integrated Reynolds stress divergence and $F$ is the bottom form stress cumulated at the level $z$ from the bottom pressure term, 


$$
F(z)=\int_{z}^{0} \sum_{\text {ridges }} \delta \bar{p}_{b} d z=\sum_{\text {ridges }} \int_{x_{W}(z)}^{x_{E}(z)} \bar{p}_{b} \frac{\partial h}{\partial x} d x
$$

Since $\phi(z=-h)=0$ the balance Eq. (7) is recovered if $R$ neglected as before, with $F(z=-h)$ as the total form stress. However, instead of the interfacial form stress balance we are now facing in the interior a balance between the integrated Coriolis force and frictional, Reynolds and bottom pressure stresses. The balance is described in Stevens \& Ivchenko (1997) for FRAM and repeated for many other eddy resolving models (see the summary in Olbers 1998). For POP we show the terms of the vertically integrated balance Eq. (10) in Fig. 15. It is obvious which terms are the main players in the different depth ranges. In the top layer these are Coriolis force and wind stress. At intermediate depths where topography is not yet intersecting there is little change in $\phi$ with depth, i.e. small meridional transport and thus small Coriolis forces balanced by small Reynolds and frictional effects ${ }^{2}$. And in the deep blocked layers the balance between Coriolis force and bottom form stress can be seen. The total balance of zonal momentum in this POP experiment is shown in Fig. 13.

Where are the eddy effects in this framework? They are hidden in the Eulerian Coriolis force, as will be discussed in Section 5 where we proceed with the Eulerian framework and include the still missing connection to thermohaline forcing and turbulent mixing.

\subsection{Failure of Sverdrup balance}

An outstanding feature of Southern Ocean dynamics is the failure of one of the cornerstones of theoretical oceanography - the Sverdrup balance $\beta \psi_{x}=$ curl $\boldsymbol{\tau}_{0}$. It relates the northward transport $V=\psi_{x}$ (the meridional velocity vertically integrated from the bottom to the surface) to the local curl of the wind stress vector $\tau_{0}$. Here, $\beta$ $=d f / d y$ is the meridional gradient of the Coriolis parameter $f$. Closure of the circulation occurs by a western boundary current to satisfy mass conservation. Apparently, in the range of latitudes of Drake Passage the Sverdrup balance must fail: $\oint V d x$ must be zero to ensure mass conservation of the piece of ocean to the south, yet the wind stress curl will not integrate to zero in general. Contrary to the circulation in an ocean basin, we cannot overcome this problem by some kind of boundary current returning the mass flux.

Nevertheless we are aware of many attempts to generate a Sverdrupian solution for the ACC. Notable is Stommel's approach (Stommel 1957) where the Antarctic Pensinsula is expanded to the north to block the Drake Passage latitude band and allow only for a northward passage. Similar barotropic theories have been presented more recently by

${ }^{2}$ Locally the Coriolis forces are large. They generate the pressure gradients which are needed to establish the interfacial form stress.
Webb (1993), Ishida (1994), and Hughes (2002). While we may classify these studies as theoretical test cases, the approach of Baker (1982) is more intriguing: in an attempt to estimate the ACC transport from wind data Sverdrup's balance is integrated along $55^{\circ} \mathrm{S}$, i.e. just north of Drake Passage in a possibly 'Sverdrupian regime', starting at the west coast of South America and extended to the east flank of the ACC system, leaving out the part where it shoots northward after leaving Drake Passage (see Figs 1 \& 2). Because of mass conservation the ACC transport running through a piece of the section that must be equal to the (negative) integrated curl of the wind stress of the remaining part - the ACC transport could then be explained entirely in terms of a certain property of the Southern Ocean wind system. In fact, for particular wind stress climatology data Baker found reasonably good agreement with the observed ACC transport.

The fallacy in Baker's approach is not in the particular choice of the integration path, it is that the Sverdrup balance is not applicable to most of the Southern Ocean (and possibly most of the world ocean, see Hughes \& De Cuevas 2001). It neglects the interaction of the circulation with the topography, which could be suspected to be important from the penetration of the ACC to great depth, unlike currents in basin gyres. In ocean basins the deep pressure gradients are shut off during spin-up of the circulation by westward propagating baroclinic Rossby waves of successively increasing vertical mode number (Anderson \& Gill 1975). In the Southern Ocean the strong and deep reaching eastward current hinders even the fastest (first baroclinic) mode from westward propagation (see e.g. Hughes et al. 1998). The establishment of deep pressure gradients not only makes the work of the bottom pressure on topography, the $B F S$, effective, it also modifies the Sverdrup theory. The Sverdrup balance derives from the planetary vorticity conservation, $\beta v=f w_{z}+\operatorname{curl} \tau_{z}$, which states that a piece of the water column which is affected by friction ( $\tau$ is the frictional stress appearing locally in the water column) or is stretched vertically must experience an appropriate advection of planetary vorticity $(\beta v=v d f / d y)$. The Sverdrup balance results by vertical integration of the vorticity balance under the assumption of vanishing vertical motion at great depth so that there is no stretching of the total water column and no friction at depth. In the presence of submarine topography and deep pressure gradients this is not valid: geostrophic flow across topography induces a vertical motion, $w_{g}(z=-h)=-\boldsymbol{u}_{g}(z=-h) \cdot \nabla h=-(1 / f) J\left(p_{b}\right.$, $h$ ), and stretching ${ }^{4}$. Hence, with the rigid lid assumption at the surface, $w(z=0)=0$, we get

$$
\beta \frac{\partial \psi}{\partial x}=\operatorname{curl}\left(\tau_{0}-\tau_{b}\right)+J\left(p_{b}, h\right)
$$

The frictional stress $\tau_{b}$ of the flow on the bottom is

\footnotetext{
${ }^{4}$ We introduce here the Jacobian operator, $J(a, b)=a_{x} b_{y}-a_{y} b_{x}$.
} 

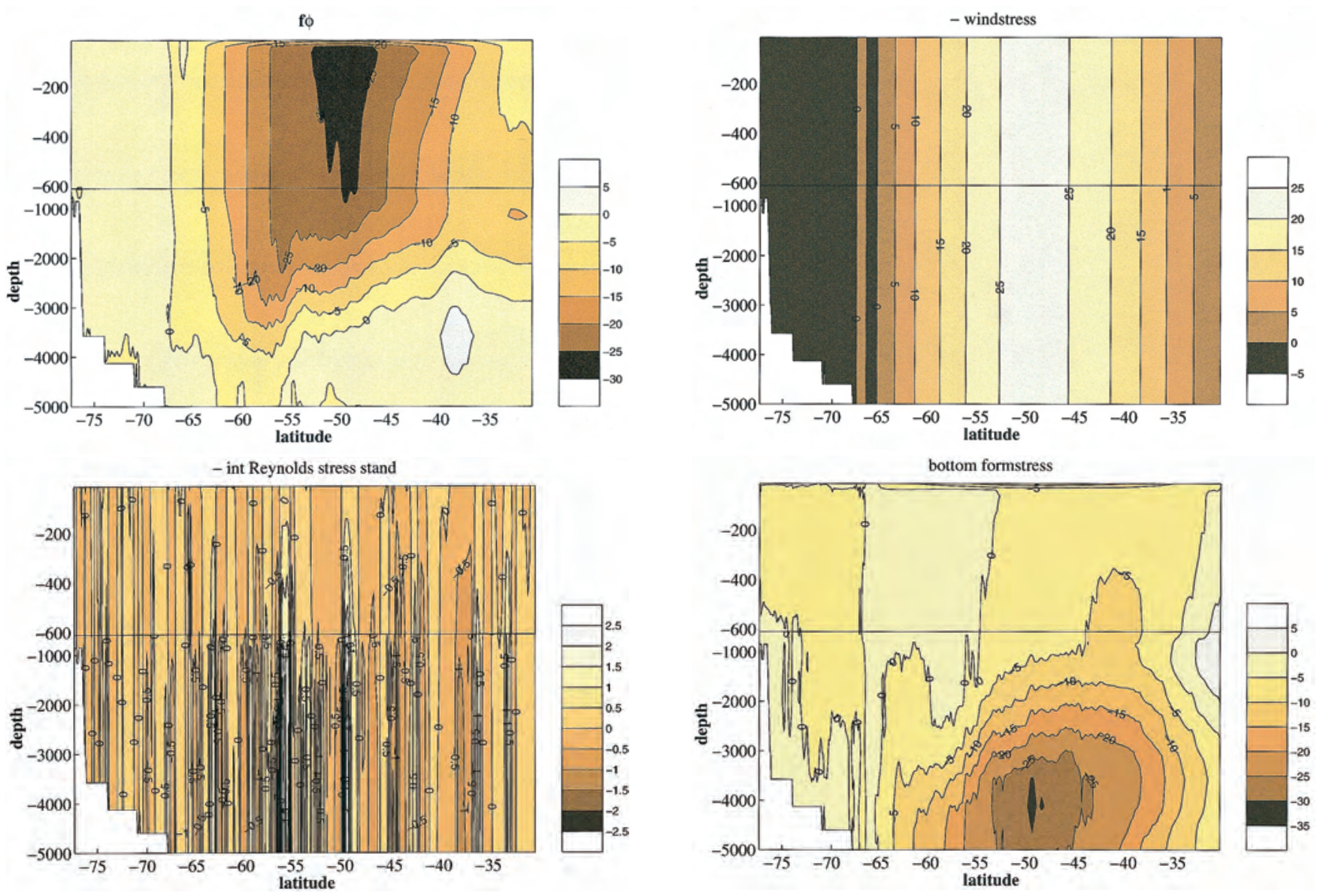

Fig. 15. Terms of the vertically integrated momentum balance (10) for the POP model as function of depth and latitude (note: the values at depth $z$ reflect the contribution to the balance of zonal momentum in the depth interval from the surface to the level $z$ ): Coriolis force $f \phi$ [upper left panel], wind stress $\left\{\tau_{0}\right\}$ [upper right panel], Reynolds stress $R$ [lower left panel], bottom form stress $F$ [lower right panel]. Units: all quantities are normalized by a mean Coriolis frequency; Contour interval is $5 \mathrm{~Sv}$ except for Reynolds stress where it is $1 \mathrm{~Sv}$. From Olbers \& Ivchenko (2001).

generally small but the so called bottom pressure (or topographic) torque $J\left(p_{b}, h\right)$ (Holland 1973) can locally be very large, even overwhelming the torque by the wind stress by an order of magnitude or more. This is demonstrated in Fig. 16 showing the streamfunction and the bottom pressure torque in a simulation with the global eddy permitting OCCAM model (Ocean Circulation and Climate Advanced Modeling Project, see Coward (1996) for details). Clearly, northward excursions of the current are correlated with positive bottom torques and southward with negative, as suggested by the barotropic vorticity balance Eq. (12). We should mention that this view applies on scales of a few degrees which are clearly larger than those of individual eddies. On smaller scales the neglected advection of relative vorticity comes into play and the dominant balance is between the bottom torque and nonlinear advection terms (Wells \& De Cuevas 1995). In any case the simple Sverdrup theory does not apply.

Notice that the zonally averaged balance of barotropic vorticity is consistent with the balance of total momentum Eq. (7): integrating Eq. (12) around a latitude circle yields the meridional divergence of Eq. (7). Though there is local compensation of the $\beta$-term and the bottom form stress as indicated in Fig. 16, the wind curl and the bottom torque balance in the zonal mean.

We should like to point out that Eq. (12) is merely a balance that the circulation has to satisfy (possibly augmented by the so far neglected terms such as lateral friction, see below). It is not sufficient to determine the streamfunction because the bottom pressure and frictional torques are not prescribed functions like the wind stress curl but rather must be determined from a complete solution. How this can be achieved is the subject of the next section.

\subsection{The geostrophic contours}

In the $\beta$-term of Eq. (12) the transport $V=\psi_{x}$ appears which is normal to latitude circles. From a mathematical point of view, latitude circles are the characteristics of the differential equation. Some of the problems discussed above arise from the periodicity of these characteristics in the latitude belt of Drake Passage. There is another vorticity 


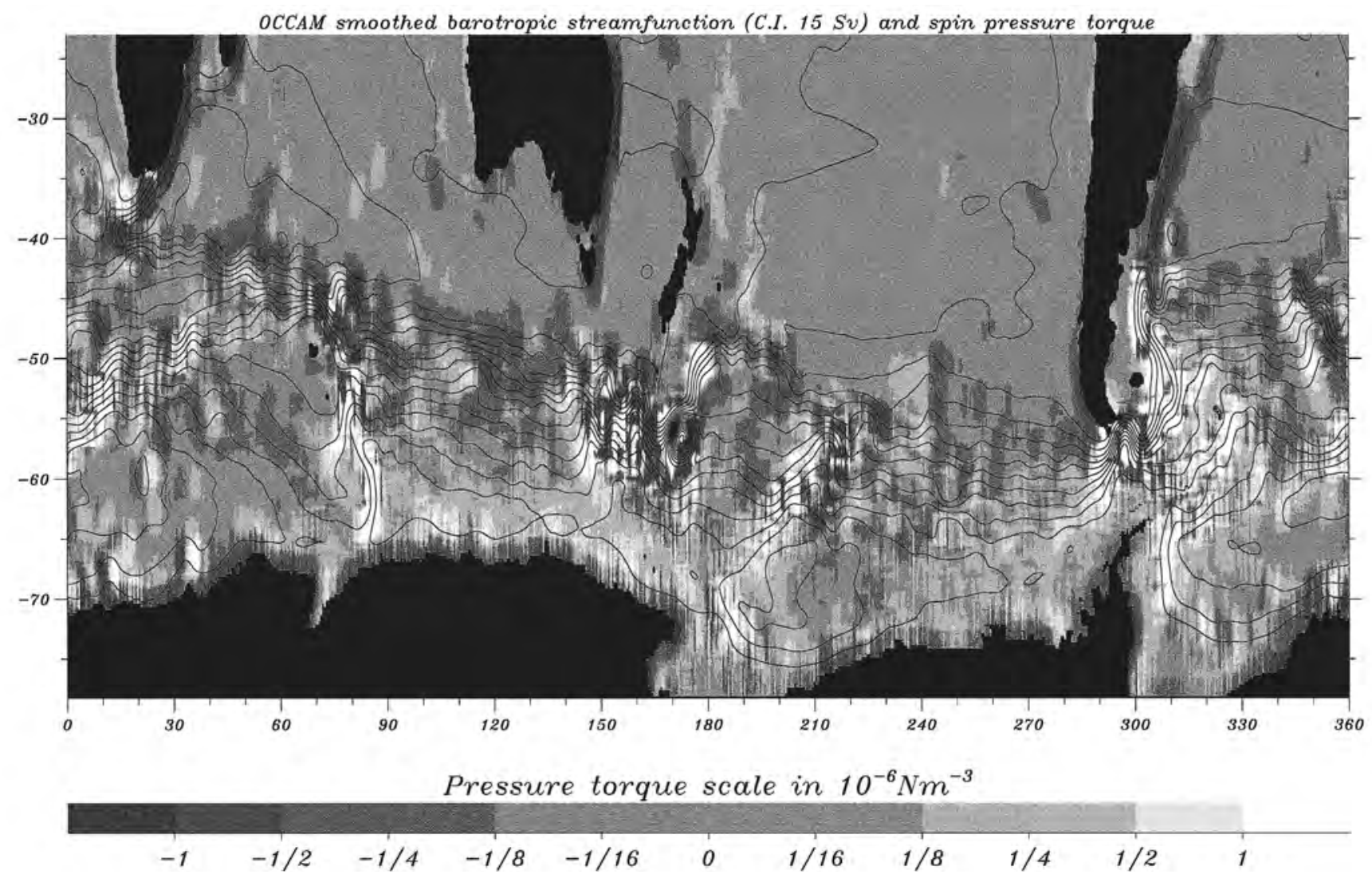

Fig. 16. Barotropic streamfunction from the OCCAM model (contour interval $15 \mathrm{~Sv}$ ) and bottom pressure torque (shading) in units of $10^{-6} \mathrm{~N} \mathrm{~m}^{-3}$. Both quantities have been smoothed by $4.25^{\circ}$ of longitude and $3.25^{\circ}$ of latitude. Note that northward flows are associated with positive torques, southward flows with negative torques. For comparison, typical wind stress curl in this region gives a torque of $10^{-7} \mathrm{~N} \mathrm{~m}^{-3}$. From Rintoul et al. (2001).

statement about the circumpolar flow with other characteristics, namely the geostrophic contours $f / h$, which in the majority do not close in themselves around Antarctica: they either run into continents or they close around topographic plateaus in the Southern Ocean (like Kerguelen) or run around the immediate rim of Antarctica (see Figs $6 \& 18$ ). The role of $f / h$ contours as characteristics becomes evident if we divide the momentum balance (3) by the depth $h$ before taking the curl. We arrive at

$$
\boldsymbol{U} \cdot \boldsymbol{\nabla} \frac{f}{h}=J\left(\psi, \frac{f}{h}\right)=J\left(\chi, \frac{1}{h}\right)+\operatorname{curl} \frac{1}{h}\left(\tau_{0}-\tau_{b}+\boldsymbol{F}\right)
$$

which identifies the agents which force transport across the geostrophic contours $f / h=$ const. These are the baroclinic torque (or the JEBAR term, Joint Effect of Baroclinicity And Relief, Sarkysian \& Ivanov 1971), and the curl of the depth averaged frictional stresses applied at the top and the bottom. We have augmented the barotropic vorticity balance by the term $\boldsymbol{F}$, indicating the effect of hitherto neglected lateral Reynolds stresses in the vertically integrated momentum balance Eq. (3). Both the lateral transport $\boldsymbol{F}$ and the bottom frictional stress $\boldsymbol{\tau}_{b}$ depend on the actual state of the flow. To make the balance more specific for later use we put in linear bottom friction and lateral diffusion of the depth integrated velocity, thus $\tau_{b}=\varepsilon \boldsymbol{U}, \boldsymbol{F}=$ $A_{h} \nabla^{2} \boldsymbol{U}$ so that

$$
\operatorname{curl} \frac{\tau_{b}}{h}=\varepsilon \nabla \cdot \frac{1}{h} \nabla \psi \quad \operatorname{curl} \frac{\boldsymbol{F}}{h}=A_{h} \nabla \cdot \frac{1}{h} \nabla^{2} \nabla \psi
$$

The advantage of Eq. (13) over Eq. (12) is obvious in homogeneous ocean: for constant density the JEBAR term drops from Eq. (13) whereas the latter would still contain the bottom torque of the barotropic (surface) pressure contained in $p_{b}$.

With wind stress and potential energy prescribed Eq. (13) is able to predict the streamfunction if suitable boundary conditions are set. Besides conditions required by the lateral friction term (usually no-slip condition for $\boldsymbol{U}$ on the coasts) we have to satisfy mass conservation, which requires $\psi=$ constant on coasts, with different constants on the different islands because these values determine the transports between them. One constant may be set to zero without restriction (e.g. $\psi=0$ on the American continent), the other constants must be predicted, which states the need for additional equations. These follow from the requirement that a solution of Eq. (13) must allow the calculation of the pressure field $p_{b}$ from the momentum balance Eq. (3) (with $\boldsymbol{F}$ included; $p_{b}$ is calculated by path integration from one 



a.

coastal point where its value may be set arbitrarily). In
multi-connected domain, with islands present, th
uniqueness of $p_{b}$ is guaranteed by the integrability
conditions
$\oint_{\text {island }} d s \cdot \nabla p_{b}=\oint_{\text {island }} d \boldsymbol{s} \cdot \frac{1}{h}\left[f \nabla \psi-\nabla \chi+\boldsymbol{\tau}_{0}-\boldsymbol{\tau}_{b}+\boldsymbol{F}\right]=0$

around each island (e.g. Antarctica) on an arbitrary path. With $n$ islands (or continents) there are thus $n-1$ such conditions which render the reconstruction of $p_{b}$ pathindependent.

The barotropic vorticity Eq. (13), together with the constraint Eq. (15), is evidently a central tool for the determination of ocean transports. The potential energy $\chi$ has to come from the baroclinic equations of heat and salt conservation where $\psi$ couples in via advection. Numerical ocean models using the rigid-lid approximation actually determine the depth-integrated velocity vector from a vorticity equation setup as Eqs (13) \& (15).

The importance of the geostrophic contours and of JEBAR were demonstrated in a series of early numerical experiments with the GFDL model of the world ocean circulation. Bryan \& Cox (1972) presented the circulation for a homogeneous fluid (constant density, thus having zero JEBAR) in an ocean with continents but of constant depth. Cox (1975) extended the studies to the cases of variable topography (with blocked $f / h$ contours but still zero JEBAR) and also to a topographic ocean with baroclinicity, hence nonzero JEBAR. Due to limited computer resources, the last experiment was largely a diagnostic simulation, i.e. the thermohaline fields do not deviate much from the initial state taken from observations. But full prognostic experiments have since then been repeated many times (e.g. Han 1984a, 1984b, Cai \& Baines 1996) with very similar results. New simulations of these cases are discussed in Olbers \& Eden (2003) and depicted in Fig. 17. They were



C.

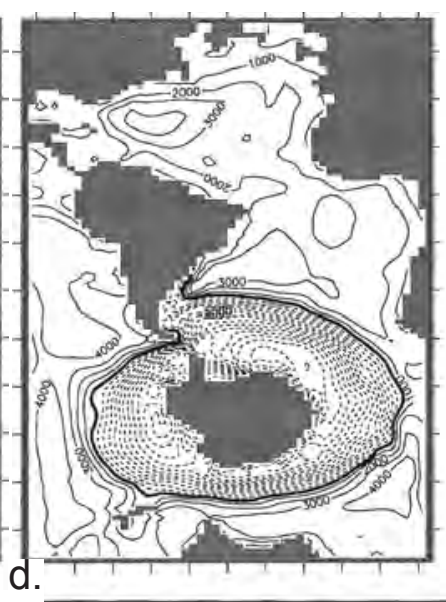

NCEP. a. streamfunction for a homogeneous flat bottom ocean, 
cannot operate, friction is the only momentum sink and with the diffusive parametrization of lateral eddy induced transports of momentum by a diffusivity $A_{h}$, as in Eq. (14), the zonal transport is proportional to $Y^{3} \tau_{0} / A_{h}$ where $Y$ is the width of the current. We are facing 'Hidaka's dilemma' (Hidaka \& Tsuchiya 1953, see Wolff et al. 1991): either we implement a reasonably sized diffusivity and then get an unrealistically large transport or we must use an unrealistically large eddy viscosity to get a reasonable size of the ACC transport.

The topographic homogeneous case has a transport that is far too low. The system now establishes a bottom form stress and a bottom pressure torque (but not JEBAR) from the surface pressure being out-of-phase with the submarine barriers of the flow (see Fig. 14). The current is mostly along the geostrophic contours which are north of the Drake Passage belt. Apparently, the frictional torques in Eq. (13) are too weak to push mass across these contours in a significant amount.

There is an interesting lesson to learn from the momentum balance Eq. (3): the component oriented along $f / h=$ const is given by

$$
\frac{\partial \psi}{\partial s}=\frac{h}{f} \frac{\partial p_{b}}{\partial s}+\frac{1}{f}\left(\tau_{0}-\tau_{b}+\boldsymbol{F}\right) \cdot \hat{\boldsymbol{s}}
$$

where $s$ is the path length coordinate along geostrophic contours and $\hat{\boldsymbol{s}}$ is the tangential unit vector. If the contours are blocked by continents (as in the Pacific sector, see Figs 6 \& 18) the transport between them is $\Delta \psi \simeq(h / f) \Delta p_{b}+V_{E k}$ where $V_{E k}$ is the Ekman transport across the contour and $\Delta p_{b}$ the pressure difference between the continents (the other friction terms are small in this regime). We may interpret this latter term as a net geostrophic transport sustained by the pressures on the coasts. On the other hand, with small or zero $\Delta \psi$ we see that the net wind stress along $f / h$-contours is taken up by a pressure difference on the continents - just as in the Sverdrup circulation regime in a flat bottom basin bounded by continents. For the $f / h$-contours closed on the rim of Antarctica we have a Hidaka-type transport regime where wind stress is balanced by friction. The transport, however, is small because the wind is much weaker (and actually westward, see Fig. 19) and the width of this region small. The closed $f / h$-regimes on the Mid-Atlantic Ridge and around Kerguelen are governed by friction as well as balancing a small net Ekman transport into these regions.

The final case which considers topography and baroclinicity gets a reasonably sized ACC transport for which clearly JEBAR is responsible. It is an order of magnitude larger than the wind curl but that property alone would not explain why this new forcing should not be blocked by the $f / h$-contours as the wind curl is blocked. In fact, the JEBAR field has a very particular spatial structure: highs and lows are placed right along the undulating path of the geostrophic contours (see Fig. 18) to help the current to circumvent the $f / h$-constraint. We may state this property in a different framework: from Fig. $17 \mathrm{c} \& \mathrm{~d}$ it appears that $\psi$ and $\chi$ are highly correlated, suggesting a functional relation $\chi=C(\psi)$ established by the dynamics. This relation is plotted in the last panel of Fig. 18. A reasonable fit is suggested from Eq. (4), thus roughly $\chi=f_{0} \psi+$ const, and this casts the vorticity balance (13) into

$$
\frac{\beta}{h} \frac{\partial \psi}{\partial x} \simeq \operatorname{curl} \frac{1}{h}\left(\tau_{0}-\tau_{b}+\boldsymbol{F}\right)
$$

The topographic $\beta$-term and JEBAR in Eq. (13) combine to achieve new (unblocked) characteristics which actually are those of the flat bottom problem.

\section{The dependence of transport on forcing}

What are the mechanisms and forcing functions that determine the transport of the zonal flow? The considerations of momentum and vorticity balances and the associated fluxes through the circulation system, outlined in the previous sections, do not answer this question. Indeed, one cannot expect a prediction of the ACC transport from just one or two integral balances. They indicate, however, that wind forcing and vertical momentum flux by transient and standing eddies and waves are important but also the processes which set up the surface and interior pressure field, which is not really helpful because it covers nearly all possible mechanisms. The baroclinic pressure aspect brings the local surface fluxes of heat and freshwater into focus (see Fig. 19). Combined as the surface density flux they determine the density field in concert with advection and diapycnal mixing - and then it might correctly be suspected that side issues such as mixing by small-scale turbulence and remotely forced agents, such as the import of NADW into the Southern Ocean, could have an influence on the ACC transport. A complete theory capable of predicting the absolute transport of the ACC is thus a formidable challenge. For quantitative answers a full model including external forces by the wind stress and the surface fluxes of density (or buoyancy) as well as the advection of mass (volume) and density must be solved, which points towards studies with numerical OGCMs. Though quite a suite of carefully designed numerical experiments exists (some have been mentioned in the previous sections and more will be discussed below) their contribution towards an understanding of the shaping of ACC transport is limited. Many of the studies have the flavour of an engineering task: changing parameters and/or forcing and monitoring the results. These provide qualitative answers but a deeper insight into the dynamics of the ACC can be obtained by cheaper methods which may reveal mechanisms in trade for completeness.

We have mentioned above the Hidaka regime, describing an entirely frictional zonal current in a flat bottom ocean with a simple transport formula. Another simple concept follows from the momentum balance in adiabatic conditions 

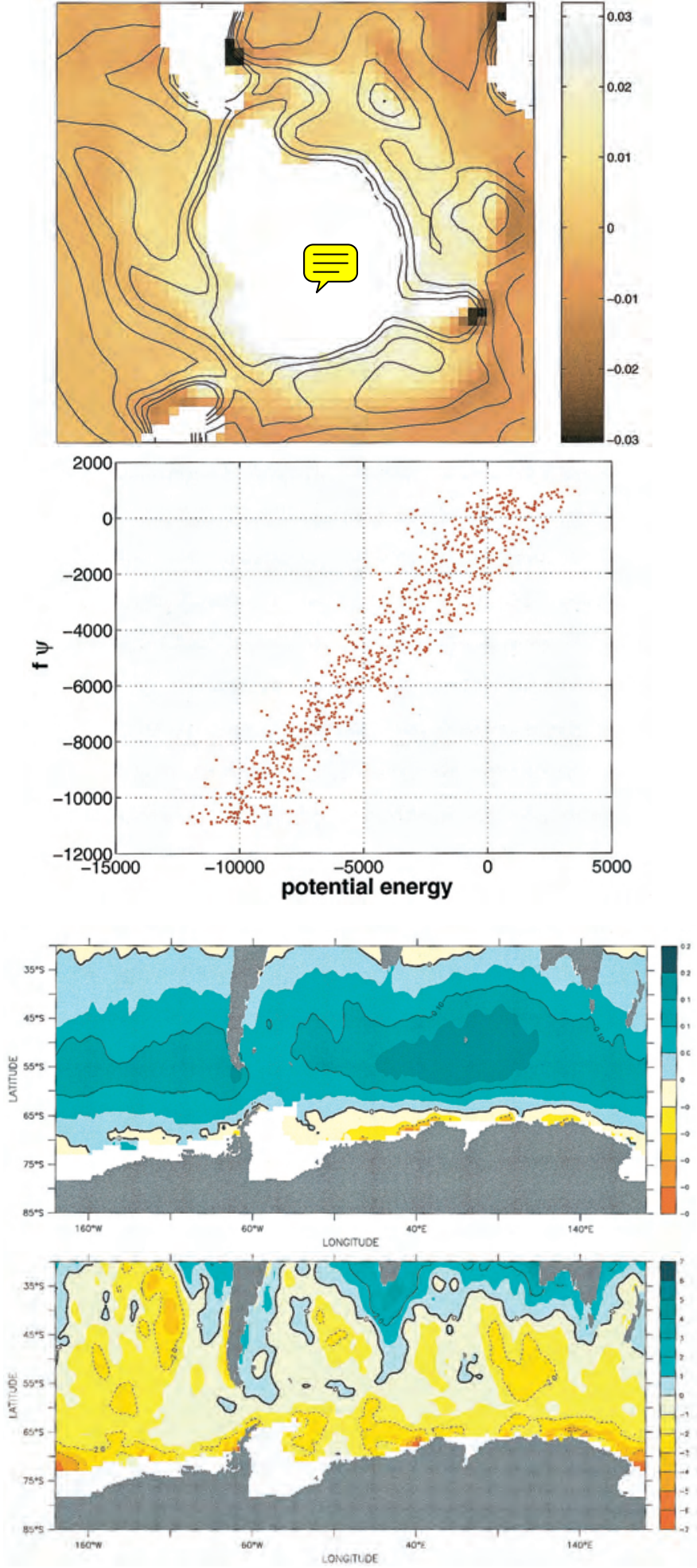

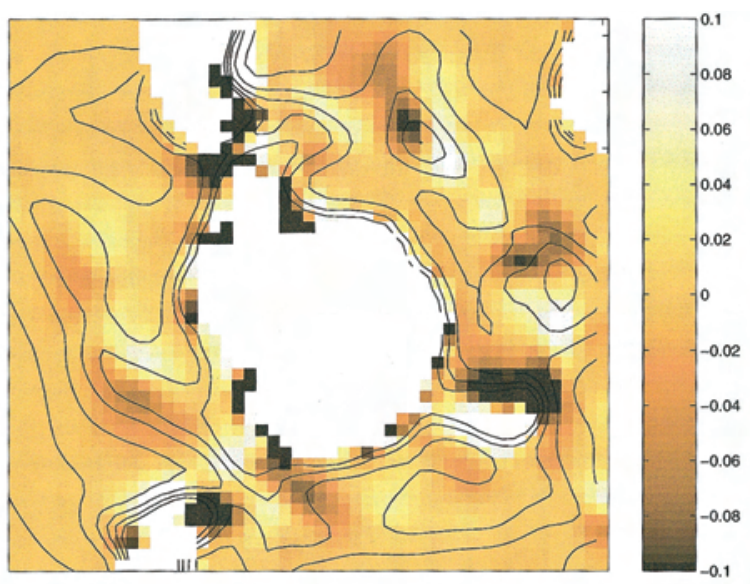

Fig. 18. Upper panels: curl of wind stress curl $\left(\boldsymbol{\tau}_{0} / h\right)[$ left $]$ and JEBAR [right] with $f / h$-contours overlaid (notice the different scale of the two plots, the colourbar for the wind curl runs from -0.03 to 0.03 , for JEBAR from -0.1 to 0.1 , units are $10^{-11} \mathrm{~s}^{-2}$ ).

Lower panel: correlation between $\psi$ and $\chi$. All for the Southern Ocean sector of the BARBI experiment c) from Fig. 17.

Fig. 19. Climatology data of zonal wind stress [upper left, unit $\mathrm{Nm}^{-2}$ ], surface heat flux [upper right, unit Wm${ }^{-2}$, and freshwater flux [lower left, unit $10^{-8} \mathrm{~m} \mathrm{~s}^{-1}$ ] and density flux [lower right, unit $10^{-6} \mathrm{~kg} \mathrm{~m}^{-2} \mathrm{~s}^{-2}$ ] (from SOC Climatology, Josey et al. 1998). The wind stress is above $0.1 \mathrm{Nm}^{-2}$ over most of the ACC region and largest in the Indian Ocean. Values for the air-sea heat exchange are controversial. In the SOC climatology the Atlantic-Indian Antarctic waters gain heat at a rate of roughly $25 \mathrm{Wm}^{-2}$ but values may be biased. The Southern Ocean gains freshwater from the atmosphere and continental runoff at a rate of roughly $10^{-5} \mathrm{~kg} \mathrm{~m}^{-2} \mathrm{~s}^{-1}$, net estimates for the Antarctic region south of $60^{\circ} \mathrm{S}$ lie around $0.25 \mathrm{~Sv}$. The flux of density is dominated by the freshwater gain: the Antarctic region looses density at the surface, thus gains buoyancy (see also Fig. 23) where surface waters are transported to the north. Closer to the coast, where winds are westward and the Ekman transport is southward a loss of buoyancy is expected (see also Fig. 12). The thick line in all figures is the zero contour. 
which results in Eq. (8). Instead of emphasizing the lateral eddy flux of momentum as in the previous flat bottom model it is based on the 'constant vertical flux scenario' (appropriate to an eddy-active ocean with no diapycnal mixing). Replacing the IFS by the lateral eddy heat or density flux as indicated in Section 3.2 we get the JohnsonBryden relation (Johnson \& Bryden 1989), $f \overline{v^{\prime} \rho^{\prime}} / \bar{\rho}_{z}=\tau_{0}$, here written for density and transient eddies (denoted by a dash). The standing eddy component is neglected (which is a severe assumption because it exceeds the transient component in realistic conditions) or - if the mean is interpreted as average along the current path - $\tau_{0}$ is not the zonal wind stress but rather the path following component (so we use dashes in the following arguments). According to the formula the 'northward' eddy density flux $\overline{v^{\prime} \rho \text { ' }}$ in the circumpolar belt of the ACC, suitably normalized, is of the size of the 'zonal' wind stress $\tau_{0}$ (so there would no meridional overturning circulation, which may rightly be questioned). In a first step Johnson \& Bryden parameterize the transient lateral eddy flux by a down-gradient form, $\overline{v^{\prime} \rho^{\prime}}=-K \bar{\rho}_{y}$, and find that the wind stress and the eddy diffusivity constrain the slope of the isopycnals, $s=-\bar{\rho}_{y} / \bar{\rho}_{z}=\tau_{0} /(f K)$. Such a relation is roughly consistent with the observed slopes in the ACC belt ${ }^{4}$ if the eddy diffusivity is of order $K \sim 10^{3} \mathrm{~m}^{2} \mathrm{~s}^{-1}$ (take $s=10^{-3}, \tau_{0}=$ $10^{-4} \mathrm{~m}^{2} \mathrm{~s}^{-2}$ ). Johnson \& Bryden (1989) proceed replacing the lateral density gradient using the thermal wind relation, $f u_{z}=g \rho_{y}$, and find

$$
K \frac{f^{2}}{N^{2}} u_{z}=\tau_{0}
$$

where the vertical density gradient is replaced by the squared Brunt-Vaisala frequency $N^{2}=-g \rho_{z}$. Apparently, $K(f / N)^{2}$ defines an equivalent diffusivity for the vertical momentum transfer which is achieved by lateral density diffusion (see e.g. Rhines \& Young 1982, Olbers et al. 1985). We see here the same equivalence between vertical momentum transfer and horizontal heat transfer by eddies as in Section 3.2. In a second step Johnson \& Bryden used Green's form (Green 1970, Stone 1972) of the diffusivity $K$ $=\alpha \ell^{2} / T$. It is obtained for a baroclinically unstable zonal current, where $\ell$ is a measure of the eddy transfer scale, and $T=N /\left|f u_{z}\right|$ is the Eady time of growth of the unstable eddies (Eady 1949). The constant $\alpha$ measures the level of correlation between $v$ ' and $\rho$ ' in the density flux ( $\alpha=0.015$ \pm 0.005 according to Visbeck et al. 1997). Johnson \& Bryden's result for the ACC transport is obtained by relating the turbulence scale $\ell$ to the baroclinic Rossby radius $\lambda=$ $N h /(|f| \pi)$. For $\ell=\pi^{2} \lambda$ we get their estimate of the shear

$$
u_{z}=\left(\frac{\tau_{0}}{\pi^{2} \alpha h^{2}} \cdot \frac{N(z)}{|f|}\right)^{1 / 2}
$$

which yields by integration the transport relative to the

\footnotetext{
${ }^{4}$ In detail there are substantial and dynamically relevant deviations from this adiabatic model, which we reconsider in Section 5.
}

bottom. The shear and thus also the transport is proportional to the square root of the wind stress. With some reasonable values of parameters a transport of about hundred $\mathrm{Sv}$ relative to the bottom can be obtained.

The Johnson-Bryden model has been much discussed as a theory of the ACC transport. Attempts to verify the squareroot relation with numerical models are plentiful (e.g. Gnanadesikan \& Hallberg 2000 with coarse-resolution models with simple geometry, Gent et al. 2001 for coarseresolution global models, Tansley \& Marshall 2001 for twolayer channel models) but generally without success. This is not surprising in view of the many assumptions put together in this model. First, there is the assumption of the adiabatic state of the flow (zero diapycnal mixing) which is violated in the real ocean but also in numerical models operating on $z$-levels (isopycnic models may be adjusted close to an adiabatic state). Second, it is not clear whether the Eady model and other details of baroclinic instability theory are appropriate in the ACC eddy field. Certainly some of these features are generated by baroclinic instability but they are not in the initial growth state but rather in some state of equilibration. Furthermore, the above parameterizations are not implemented in most coarse OGCMs. Even eddy fluxes deduced from eddy resolving models show a quite poor agreement with eddy flux parameterizations (Bryan et al. 1999 and Olbers \& Ivchenko 2001 for the POP model). Finally, we might also expect that the stratification, entering in the Johnson-Bryden concept only in a prescribed $N(z)$, would at least partly be set by the action of the wind and the overturning circulation, to point here again at the missing thermohaline forcing. This is convincingly demonstrated by Gnanadesikan \& Hallberg (2000) with simple models in which the buoyancy forcing has a direct feedback on the density structure (the tilt of the interface in a two-layer ocean). The wind stress and buoyancy feedback interplay in a complex way via the balance of northward Ekman transport and the upwelling through the thermocline to produce a meridional pressure gradient across the unblocked latitudes which is in balance with the baroclinic part of the ACC transport. The net transport is then clearly not uniquely determined by the wind stress.

The above concepts miss the influence of topography. The total momentum balance Eq. (7) contains the part of the bottom pressure which is out of phase with variations of the topography along the zonal path of integration. Some insight into the mechanism which the flow uses to generate bottom form stress has been gained from heavily truncated images of the full dynamics, so called low-order models where the flow fields are represented by very few spectral components (hopefully those of dynamical relevance, see Olbers 2001). In the barotropic Charney-DeVore model (Charney \& DeVore 1979, see also Olbers \& Völker 1996, Völker 1999) the topography is taken sinusoidal in the zonal direction: if topography is sine, form stress is cosine. The model resolves the zonal current $u$ and the sine and cosine 
components of pressure. The latter are established by a standing barotropic Rossby wave which is generated by the mean flow $u$ going over the topography. At the upstream side of the hills the fluid must be lifted up thus making high pressure, at the downstream side a pressure low follows. The naturally westward propagating wave becomes stationary by eastward advection in the zonal current and friction: it is locked in resonance with the mean flow and produces a form stress which becomes a nonlinear functional of the zonal velocity,

$$
B F S[u]=-\frac{1}{2} \varepsilon u h \frac{(f \delta)^{2}}{\varepsilon^{2}+k^{2}\left(u-c_{R}\right)^{2}}
$$

Here $k$ is the zonal wavenumber of the topography, $\delta$ the ratio of height of the hills above the ocean floor to the mean depth, $c_{R}=\beta / k^{2}$ the speed of barotropic Rossby waves and $\varepsilon$ a parameter of linear bottom friction, $\tau_{b}=-\varepsilon u h$. The total momentum balance (7), written now as $\tau_{0}-\varepsilon u h+B F S[u]=$ 0 , then determines the zonal transport $u h$. Three equilibria are found if $\tau_{b}(h \varepsilon)$ is well above $c_{R}$, two are stable circulation regimes. For the two solutions in the resonant range ( $\mathrm{u}$ close to $c_{R}$ ) the friction in the momentum balance is negligible, these solutions are balanced by form stress. The off-resonant solution is controlled by friction. It is remarkable that friction is essential in all cases to shift the pressure field out-of-phase with respect to the topography.

Charney \& DeVore (1979) have developed this model for atmospheric flow regimes. In the ocean the resonant solutions do not exist - flow speeds are much less than speeds of barotropic Rossby waves - and reasonable values for the wind stress and the bottom friction allow only for the frictionally controlled solution

$$
u h=\frac{\tau_{0} / \varepsilon}{1+\delta^{2}(a k)^{2} / 2} \quad B F S[u]=-\frac{1}{2} \varepsilon u h \delta^{2}(a k)^{2}
$$

where $a=|f / \beta|$ is the earth radius times tangent of latitude. The transport in this barotropic model decays away from the frictional solution $u h=\tau_{0} / \varepsilon$ (with hundreds $\mathrm{Sv}$ transport) with increasing height of the topography. The drag of the form stress increases quadratically with the height of the topography and attains higher values than friction for moderately sized submarine ridges. In a baroclinic extension the Charney-DeVore resonance can operate in realistic ACC conditions. This will be discussed in Section 4.2 .

We should mention that the above model works on an infinite $\beta$-plane but also in a zonal channel - a set-up which might be more appropriate for the ACC. However, here the $f / h$ contours become blocked at some critical topography height $\left(\delta>\delta_{c}=2 \mathrm{Y} /(\pi|\mathrm{a}|), Y=\right.$ channel width) and then the flow regime is not well represented by a few low-order modes (see Olbers et al. 1992). In fact, analytical and numerical solutions (Krupitsky \& Cane 1994, Wang \& Huang 1995) of barotropic channel flow reveal a current regime which is entirely unrealistic for application to the ACC: the flow is in narrow frictional boundary layer currents at the walls, switching side from south to north in a narrow internal layer along a connecting $f / h$-contour. In this blocked regime the flow is weak due to substantial drag by form stress and the transport is independent of friction.

In the rest of this section we extend the Johnson-Bryden concept to include the missing thermohaline forcing, and present a linear wave barotropic-baroclinic theory of the establishment of the bottom form stress.

\subsection{Extended Johnson-Bryden type models}

We proceed with the zonally averaged Eulerian model of Section 3.3 to extend the Johnson-Bryden concept towards the missing thermohaline component. The vertical eddyinduced flux of momentum enters via the TEM theory (Transformed Eulerian Mean, see Andrew et al. 1987, McIntosh \& McDougall 1996) in which Eq. (10) is augmented by a correspondingly averaged balance of potential density $\rho$. Assuming stationary conditions we start with the density balance $(\rho u)_{x}+\nabla \cdot \rho \boldsymbol{v}=-I_{x}-\nabla \cdot \boldsymbol{J}$ where $(u$, $v)$ is the three-dimensional velocity, $(\mathrm{I}, \boldsymbol{J})$ is the small-scale turbulent flux of $\rho$, and $\nabla$ the $(y, z)$ derivative. We separate density and velocity into a zonal mean part and deviation, e.g. $\rho=\{\rho\} / L+\rho^{*}$. As in Section 3.3 the curly bracket operator denotes zonal integration on level surfaces and $L$ is the path length. The balance of mean density $B=\{\rho\} / L$ is then obtained by zonal integration of the density balance and expressed by

$$
J(\phi, B)=-\frac{\partial}{\partial y}\left\{v^{*} \rho^{*}\right\}-\frac{\partial}{\partial z}\left\{w^{*} \rho^{*}\right\}-\nabla \cdot \boldsymbol{J}
$$

where $\phi$ is the Eulerian overturning streamfunction, $\{v\}=$ $-\partial \phi / \partial z,\{w\}=\partial \phi / \partial y$. The eddy density flux is treated as follows: the flux vector is split in the components oriented at the isopycnal, $\left(\left\{v^{*} \rho^{*}\right\},\left\{w^{*} \rho^{*}\right\}\right)=-\phi_{e d}\left(-B_{z}, B_{y}\right)-K_{d i a}\left(B_{y}\right.$, $B_{z}$ ), which introduces a diapycnal eddy-induced diffusivity ${ }^{5}$ $K_{d i a}$ and an eddy-induced streamfunction $\phi_{e d}$ given by

$$
\phi_{e d}=\frac{\left\{v^{*} \rho^{*}\right\} B_{z}-\left\{w^{*} \rho^{*}\right\} B_{y}}{|\nabla B|^{2}} \quad K_{\text {dia }}=-\frac{\left\{v^{*} \rho^{*}\right\} B_{y}+\left\{w^{*} \rho^{*}\right\} B_{z}}{|\nabla B|^{2}}
$$

This allows Eq. (23) to be rewritten as

$$
J\left(\phi_{\text {res }}, B\right)=\nabla \cdot K_{\text {dia }} \nabla B-\nabla \cdot \boldsymbol{J}
$$

The mean density is advected by a combination of the Eulerian current and the eddies with $\phi_{r e s}=\phi+\phi_{e d}$ which is called residual streamfunction. No approximation has been made yet, Eqs (23) \& (25) are identical. According to the common belief, however, the diapycnal flux of density by eddies is small and so we neglect the first term on the rhs of Eq. (25). Eliminating then the Eulerian streamfunction from

\footnotetext{
${ }^{5}$ Since the curly bracket is zonal integration rather than average the diffusivity gets the dimension $\mathrm{m}^{3} \mathrm{~s}{ }^{1}$.
} 
the momentum balance we get the TEM model

$$
\begin{aligned}
f \phi_{\mathrm{ed}}-f \phi_{\mathrm{res}} & =\left\{\tau_{0}\right\}-\{\tau\}-R+F \\
J\left(\phi_{\mathrm{res}}, B\right) & =-\nabla \cdot \boldsymbol{J}
\end{aligned}
$$

The close correspondence of this momentum balance and the isopycnal form (6) becomes obvious when we use $K_{d i a} \equiv$ 0 to write the eddy streamfunction as $\phi_{e d}=-\left\{v^{*} \rho^{*}\right\} / B_{z}$ which is the 'heat flux equivalent' of the interfacial form stress, as outlined in Section 3.3. Consequently we may view Eq. (26) as extension of the Johnson-Bryden model Eq. (8). With the residual streamfunction it includes the thermohaline part, it also includes Reynolds and bottom form stresses. We shall use the model Eq. (26) below in Section 5 to deduce the density field and the residual streamfunction (the overturning circulation) from the forcing of the system by wind and surface buoyancy flux. Here we attempt to infer from Eq. (26) the magnitude of the zonal transport.

We neglect subgrid and Reynolds stresses (which are small) and the standing eddy term (which is small if the mean is ACC path following, as discussed before) and use a downgradient parametrization of the transient eddy flux, $\left\{v^{\prime} \rho^{\prime}\right\}=-K L B_{y}$, by which $\phi_{e d}=-L K B_{y} / B_{z}=L K s$ below the mixed layer. The residual streamfunction is inferred from (26), and $\phi_{\text {res }}$, taken just below the surface mixed layer, relates to the surface density flux $\mathcal{H}_{0}$ by $\phi_{\text {res }} B_{y}=\mathcal{H}_{0}$. The two balances in (26) then lead to the relation

$$
K s-\frac{\tau_{0}}{f}=\frac{\mathcal{H}_{\mathrm{O}}}{L B_{y}}
$$

just below the mixed layer base. A similar relation is used by Speer et al. (2000) to examine transformation of watermasses around Antarctica. Assuming that mixing by turbulence is small in the interior the slope at the mixed layer base is related by Eq. (26) to that at greater depth and $K s-\tau_{0} / f=$ const on an isopycnal (see also equation Eq. (43) below). Thus, the relation Eq. (27) holds in the interior as well but the terms on the lhs are taken at the respective latitude and depth and the rhs at the corresponding isopycnal outcrop to the south. If the meridional gradient of density in the surface layer is known the complete interior density field can in fact be determined from $\tau_{0}$ and $\mathcal{H}_{0}$. Marshall \& Radko (2003) use Eq. (27) in this 'diagnostic' mode ( $B_{y}$ at the surface is given from observations) to infer the structure of the overturning circulation in the Southern Ocean.

Another diagnostic form of Eq. (27) is achieved by the assumption that the vertical gradient $B_{z}$, or the BruntVaisala frequency $N^{2}=-g B_{z}$, is known from observations. Replacing then the meridional gradient by the vertical current shear, $f u_{z}=g B_{y}$, we recover the Johnson-Bryden model in an extended form,

$$
K \frac{f^{2}}{N^{2}} u_{z}^{2}=u_{z} \tau_{0}-\frac{g \mathcal{H}_{\mathrm{O}}}{L}
$$

It now includes thermohaline forcing. If the wind stress term dominates over the surface density flux term ${ }^{6}$ the relation Eq. (19) is recovered. Under weak wind stress conditions we find a buoyancy-driven zonal flow (if the surface density flux $\mathcal{H}_{0}$ is negative, as actually found over most of the ACC, see Figs $19 \& 23$ ). Implementing the Green-Stone parametrization into the first case yields the above discussed square-root dependence of transport on the wind stress, and the second case leads to a cubed-root dependence of transport on the buoyancy forcing $-g \mathcal{H}_{0}$.

We would like to clarify that the above analysis is not a complete transport theory. Neither $B_{y}$ in the surface layer nor the profile of $N^{2}$ can be regarded as universally given parameters, as they clearly will depend on the forcing $\tau_{0}$ and $\mathcal{H}_{0}$. Equation (27), though valid over a large depth range if internal turbulence can be neglected, is not sufficient to completely determine the density field or the current profile from the forcing functions and universal parameters. An additional relation is needed, for example an equation which determines the meridional profile of the slope $s$ or of the residual streamfunction $\phi_{\text {res }}$ beneath the mixed layer from the forcing. This means that somewhere in the overturning circulation diffusion and mixing must come into play mathematically speaking a non-local problem has to be solved (the complete problem is elliptical, see Section 5). The shortcut described by Karsten et al. (2002) and Karsten \& Marshall (2002) where $\phi_{\text {res }}$ is simply set equal to the Eulerian transport $\tau_{0} / f$ (or a specified fraction of it) lacks physical grounds. A similar restriction is found in Bryden \& Cunningham (2003): in their considerations the residual transport is entirely ignored.

\subsection{The shaping of bottom form stress}

With the presentation of the barotropic Charney-DeVore model we have highlighted the role of long Rossby waves (with wavelength of the underlying topography) in shaping the bottom form stress. We have pointed out that the resonant behavior in the model cannot occur in a barotropic ocean because barotropic waves are too fast. But we can have such a mechanism in a baroclinic set-up: baroclinic oceanic Rossby waves have a speed comparable to zonal velocities in the ACC. In a baroclinic version of the Charney-DeVore model with ACC conditions Olbers \& Völker (1996) and Völker (1999) show that baroclinic waves are generated in resonance with the topography and become stationary when the barotropic current speed equals the baroclinic Rossby wave speed. The transport decreases strongly with increasing topography height, starting from a frictionally controlled state at low heights, followed by a transition to a complex resonant regime with multiple equilibria at intermediate heights, and further to a state controlled by barotropic and baroclinic bottom form stress

$\overline{{ }^{6} \text { With } \mathcal{H}_{0}=5 \times 10^{-9} \mathrm{~m}^{2} \mathrm{~s}^{-3}, Y=2000 \mathrm{~km}, \Delta B=5 \times 10^{-3} \mathrm{~ms}^{-2} \text { strong wind }}$ stress is $\tau_{0} \gg 2 \times 10^{-4} \mathrm{~m}^{2} \mathrm{~s}^{-2}$. 



Fig. 20. Model results from the primitive equation channel model for configuration SIN1 [left panels] and SIN2 [right panels] (for description see text). The three columns show experiments with horizontal eddy viscosity $10^{4} \mathrm{~m}^{2} \mathrm{~s}^{-1}$. The top row shows the barotropic streamfunction $\psi$, the second row shows the baroclinic potential energy $\chi$ divided by a mean Coriolis frequency $f_{0}$, the third row shows a zonal section of the potential temperature in the center of the channel. From Borowski (2003). 



Fig. 21. Terms of the zonal momentum balance in the channel experiments SIN1 and SIN2 of Borowski (2003), as a function of the horizontal eddy viscosity [dotted: friction, dash-dotted: wind stress, dashed: baroclinic form stress, full: barotropic form stress].

at high topography. Within the limits of such a simple model this latter regime would be appropriate to the ACC. Though the momentum balance Eq. (7) seems to operate here without friction, it is important that the phase shifts of the topographically induced pressure gradients with respect to the topographic undulations are proportional to the coefficients of bottom and interfacial friction of the model, in close correspondence to the barotropic model as given by Eqs (21) \& (22). The baroclinic topographic resonance theory determines the transport in adiabatic models in a manner similar to the barotropic Charney-DeVore mechanism: the bottom form stress is a complicated resonance function of the barotropic and baroclinic velocities and the transport follows from Eq. (7) and a corresponding balance for the baroclinic momentum. The structural properties of this low-order model are preserved when the degrees of freedom are increased from the simplest nontrivial model with 11 modes to a number representing a moderately resolved coarse model (with 75 modes). We return to such a low-order model in more detail below.

There have been numerous numerical studies with a realistic ACC configuration with coarse resolution models (Olbers \& Wübber 1991, Cai \& Baines 1996, Gnanadesikan \& Hallberg 2000, Gent et al. 2001, Borowski 2003) which investigated the dependence of transport on the buoyancy forcing at the surface, however, without revealing a clear concept of how the transport depends on the forcing functions. Distinguishing between the pressure forces generated by the topographic resonance mechanism or by a thermohaline forcing with zonal variations is non-trivial. It must be kept in mind that a forcing by a restoring term, say $\gamma\left(T_{o b s}(y)-T\right)$ for temperature, will always give rise to a nonzonal surface flux if the flow and thus $T$ are non-zonal. Borowski (2003) presents a large number of channel experiments with a numerical primitive equation model (MOM with $1^{\circ}$ meridional x $2^{\circ}$ zonal grid and 16 levels) studying the sensitivity to forcing and frictional parameters. The forcing fields (wind stress and $T_{o b s}$ ) are strictly zonal but restoring is used for temperature. Figure 20 displays the streamfunction, potential energy and a zonal section of temperature through the middle of the channel for two experiments which differ only in the restoring surface temperature $\left(10^{\circ} \mathrm{C}\right.$ gradient across the channel for experiments SIN1, $20^{\circ} \mathrm{C}$ for SIN2). However, the momentum balance is drastically different (see Fig. 21): in SIN1 the baroclinic form stress drives the eastward flow (clearly visible in Fig. 20: as in Fig. 11 the water on the western slope is lighter compared to the eastern slope), in SIN2 it decelerates (here the lighter water is on the eastern slope), in both cases the baroclinic form stress is in opposition to the barotropic form stress. It seems that the stronger gradient of surface restoring temperature puts the system into a different regime by implementing a stronger non-zonal thermal forcing.

Analytical theories of the ACC which include baroclinicity are mostly done in the quasi-geostrophic framework and use a modal truncation: the layer streamfunctions are represented by a set of structure functions for the $(x, y)$-dependence so that the dynamical equations are written only as ordinary differential equations for the time dependence of amplitudes. If the system is truncated to a few modes, a so called 'low-order model' is obtained and steady states can be investigated analytically using the mathematics of dynamical system theory or by use of numerical bifurcation tools (Dijkstra 2000). The Charney-DeVore-type models discussed above are of such kind. Here, we extend the model of Völker (1999) by adding thermohaline forcing and implementing lateral diffusion of momentum (more details are given in Borowski 2003). A two-layer zonal channel with quasigeostrophic dynamics is considered, with a sine-shaped topography in the zonal direction. The two streamfunctions are expressed by suitably chosen sinusoidal structure functions. After some eliminations the resulting system is written in terms of six amplitudes representing the barotropic transport $T$ (upper layer plus lower layer transport), the baroclinic transport $S$ (upper layer minus lower layer transport), and respective barotropic and baroclinic sine and cosine components, $T_{s}, S_{s}$ and $T_{c}, S_{c}$ (more accurately, the $T$ and $S$ quantities are based on barotropic and baroclinic velocities). The latter generate the barotropic and baroclinic form stress, respectively. It is clear that with such an enormous reduction, transient eddies are not present. Their effect on the downward transfer of momentum - the interfacial form stress IFS - is parameterized by friction acting on the interface of the two 
layers with a coefficient $\kappa$. The physical mechanism of this friction is completely equivalent to a diffusion of layer thickness (see Gent \& McWilliams 1990, Gent et al. 1995). In addition we have lateral diffusion of momentum with a viscosity $\varepsilon$. We have scaled these variables ${ }^{7}$ and the dimensionless form of the set of differential equations becomes

$$
\begin{aligned}
& \frac{\partial T}{\partial t}=W_{0}+\delta\left(T_{c}-h S_{c}\right)-\varepsilon T \\
& \frac{\partial S}{\partial t}=\frac{\lambda^{2} W_{0}}{h}-\frac{\lambda^{2}}{1-h} \delta\left(T_{c}-h S_{c}\right)+\ell\left[T_{c} S_{s}-T_{s} S_{c}\right]+\frac{Q_{0}}{1-h}-\left(\kappa+\lambda^{2} \varepsilon\right) S \\
& \frac{\partial T_{c}}{\partial t}=\beta T_{s}-2 \delta(T-h S)-m\left[T T_{s}+h(1-h) S S_{s}\right]-\varepsilon T_{c} \\
& \frac{\partial S_{c}}{\partial t}=\beta \lambda^{2} S_{s}+\frac{2 \lambda^{2}}{1-h} \delta(T-h S)-n\left[T S_{s}-S T_{s}\right]-\left(\kappa+\lambda^{2} \varepsilon\right) S_{c} \\
& \frac{\partial T_{s}}{\partial t}=-\beta T_{c}+m\left[T T_{c}+h(1-h) S S_{c}\right]-\varepsilon T_{s} \\
& \frac{\partial S_{s}}{\partial t}=-\beta \lambda^{2} S_{c}+n\left[T S_{c}-S T_{c}\right]-\left(\kappa+\lambda^{2} \varepsilon\right) S_{s}
\end{aligned}
$$

The forcing appears in $W_{0}$ (wind stress) and $Q_{0}$ (external buoyancy flux). Terms derived from nonlinear advection are found in the cornered brackets $(\ell, m, n$ are numerical coupling coefficients depending only on the channel dimensions, see footnote). The respective term in the zonal baroclinic balance Eq. (30) is the interfacial form stress (IFS) induced by the standing eddies. The $\mathrm{\kappa}$-term in that equation is the corresponding transient eddy interfacial form stress. The baroclinicity enters via the internal Rossby radius $\lambda$, and the topography height is $\delta$. Lateral friction operates in the barotropic $T$-equations and interfacial friction in addition in the baroclinic $S$-equations. The $W_{0^{-}}$ term in the baroclinic Eq. (30) arises from the Ekman pumping acting on the background stratification. The sine and cosine Eqs (31) to (34) describe planetary-topographic Rossby waves with the same zonal wave number as the topography; $\beta$ is a scaled planetary coefficient $d f / d y$. There are terms arising from nonlinearities (advection) and diffusion. Note that Eq. (29) is the balance of vertically integrated momentum, and is congruent to Eqs (7) \& (10).

\footnotetext{
${ }^{7}$ The scaling and coefficients are as follows: All transport variables are scaled by $\pi^{2} /\left(\left|f_{0}\right| Y^{2}\right)$ to yield dimensionless $T, S, T_{c}, \ldots$ Parameters are $b$ $=Y /(\pi L), \varepsilon=\pi^{2} A_{h} /\left(b\left|f_{0}\right| Y^{2}\right), \kappa=\pi^{2} K /\left(b\left|f_{0}\right| Y^{2}\right), \lambda=\pi R / Y, \beta=2 Y d f / d y /\left|f_{0}\right|$ where $L$ is the zonal length and $Y$ the width of the channel. $R=\sqrt{ }\left(g^{\prime} / f_{0}^{2}\right)$ $H_{1} H_{2} / H$ is the internal Rossby radius of a 2-layer fluid with densities $\rho_{l}$, $\rho_{2}$, mean layer thicknesses $H_{1}, H_{2}, H=H_{1}+H_{2}$ and reduced gravity $g^{\prime}=$ $g\left(1-\rho_{1} / \rho_{2}\right)$, furthermore $h=H_{1} / H$ is a thickness ratio. Coupling coefficients are $\ell=3 \pi^{2} \mathrm{~b}^{2} / 8, m=64 \pi^{2} / 3, n=16 / 3$. The scaled depth is $1+$ $(\delta / \pi) \sin (2 \pi x / L)$, thus $\delta / \pi$ is the relative height of the topography. The forcing amplitudes are $W_{0}=(3 / 16) \pi^{2} \tau_{0} /\left(b H Y f_{0}{ }^{2}\right), Q_{0}=(3 / 32) \pi^{3}$ $B_{0} /\left(b\left|f_{0}\right|^{3} Y^{2}\right)$ in terms of the zonal wind stress $\tau=\tau_{0} \sin ^{2}(\pi y / Y)$ and the surface buoyancy flux $B=B_{0} \cos (\pi y / Y) \sin (\pi y / Y)$. Here, $\tau$ and $\mathrm{B}$ are dimensioned $\mathrm{m}^{2} \mathrm{~s}^{-2}$ and $\mathrm{m}^{2} \mathrm{~s}^{-3}$, respectively.)
}

We can identify the barotropic and baroclinic contributions to the bottom form stress.

Considering the steady state of the above model we recover the most important physical mechanisms which we have outlined in this article.

The barotropic form stress drag: a barotropic state is obtained for $\lambda=Q_{0}=0$. Then all $S$-fields are identically zero and a barotropic solution as described by Eqs (21) \& (22) emerges in which the transport decays quadratically with increasing topography height due to the drag of the barotropic form stress.

Johnson-Bryden dynamics: if lateral friction is small and the influence of topography and standing eddy IFS are ignored we regain from Eq. (30) the Johnson-Bryden state Eq. (28),

$$
\frac{\kappa}{\lambda^{2}} S=\frac{W_{0}}{h}+\frac{Q_{0}}{\lambda^{2}(1-h)}
$$

in which the transport contained in the shear current is governed by wind stress and buoyancy flux.

The compensation of barotropic and baroclinic form stresses: for strong stratification (large $\lambda$ ) or vanishing nonlinearities (cornered bracket terms neglected, i.e. no advection) we learn from the zonal mean baroclinic balance Eq. (30) that if the form stress terms $\delta T_{c}$ and $\delta h S_{c}$ are individually increasing with topography height they must compensate since the remaining terms in the balance do not increase. The reason for the compensation of the barotropic and baroclinic form stresses, discussed in detail in Section 3.3 , is thus found in the compensation of the vertical lifting or lowering of the background stratification (given by $\lambda^{2}$ ) by the pumping induced by the Ekman velocity and the barotropic and baroclinic vertical velocities of the flow (the first and second terms on the rhs of Eq. (30)), the latter two being generated by the flow passing across the topography (they are proportional to $\delta$ ). It is not clear yet, however, which of the two form stresses drives and which brakes the zonal flow and under what circumstance they would increase with height of the barriers.

Breaking of 'Hidaka's dilemma' by stratification and eddies: adding Eqs (29) \& (30) in a suitable way to eliminate the form stress parts we find the balance of upper layer transport $T+(1-h) S$,

$$
\frac{W_{0}}{h}+\frac{Q_{0}}{\lambda^{2}}-\varepsilon(T+(1-h) S)=\frac{1-h}{\lambda^{2}}\left(\kappa S+\ell\left[T_{c} S_{s}-T_{s} S_{c}\right]\right)
$$

and eliminating the wind forcing between Eqs (29) \& (30), we get the balance

$$
-\frac{h Q_{0}}{(1-h) \lambda^{2}}+\frac{\delta}{1-h}\left(T_{c}-h S_{c}\right)-\varepsilon(T-h S)=-\frac{h}{\lambda^{2}}\left(\kappa S+\ell\left[T_{c} S_{s}-T_{s} S_{c}\right]\right.
$$

for the lower layer transport $T-h S$. On the rhs we find the $I F S$ due to transient and standing eddies.

Suppose for the moment that the IFS are small or the 
Rossby radius is large (strong stratification). We then arrive at the physically intuitive statement that the transport in the surface layer, $T+(1-h) S$, is decoupled from the topography: it is given by the wind stress and buoyancy flux acting in the above combination against lateral friction. The surface layer transport is then in a 'Hidaka'-type state (inversely proportional to the eddy viscosity $\varepsilon$ and thus large for reasonably sized $\varepsilon$ ). The transport of the lower layer, $T$ $h S$, is governed by the magnitude of the bottom form stress and external heating $Q_{0}$. This Hidaka dilemma must be resolved by weak stratification and large IFS from transient or/and standing eddies. Notice that the crucial term $\left[T_{c} S_{s}\right.$ $\left.T_{s} S_{c}\right]$ of the standing eddies is nullified for the flat bottom wave solution of the Eqs (31) to (34). It is, however, supported by linear topographic waves. The wave equations yield after some manipulations

$$
T_{c} S_{s}-T_{s} S_{c}=-4(\delta / \beta)^{2} \frac{\kappa}{\beta \lambda^{2}} \frac{(T-h S)^{2}}{\left((\varepsilon / \beta)^{2}+1\right)\left((\vartheta / \beta)^{2}+1\right)_{(38)}}
$$

The standing wave IFS is - in this approximation - negative if the topography is undulated and the bottom flow nonvanishing, it must transfer eastward momentum downward. It is worth noticing that it depends marginally on the viscosity $\varepsilon$ but is entirely supported by the interfacial friction $\kappa$.

The form stress terms $\delta T_{c}$ and $\delta h S_{c}$ arise by the response of the barotropic and baroclinic wave system, described by the four Eqs (31) to (34), to the zonal flow crossing the topography (there is no direct forcing because the external heating function was assumed strictly zonal). A reasonable solution of the model should yield transports in the range $\beta » T, S \approx \beta \lambda^{2}$, meaning that the flow velocities are much less than the speeds of barotropic Rossby waves but supercritical with respect to the baroclinic waves, as in ACC conditions. Then the nonlinearities in Eqs (31) \& (33) are small and these equations represent a linear barotropic planetary-topographic wave. The barotropic form stress $\delta T_{c}$ can thus be explained by long linear Rossby waves generated by the deep current, $T$ - $h S$ in the above model, crossing the large ridges blocking the circumpolar path of the ACC. In contrast, the baroclinic form stress $\delta h S_{c}$ might be governed by a nonlinear response, according to $T, S \approx$ $\beta \lambda^{2}$ in (32) and (34). We discuss the consequences further below.

Still it is worth considering the linearized version of the above model.

\subsection{A linear transport model}

We thus neglect the standing eddy IFS in Eq. (30) and the advection terms in the wave equations. The form stress terms become

$$
\delta T_{c}=-2(\delta / \beta)^{2} \frac{\varepsilon(T-h S)}{(\varepsilon / \beta)^{2}+1} \quad \delta h S_{c}=2(\delta / \beta)^{2} \widetilde{h} \frac{\vartheta(T-h S)}{(\vartheta / \beta)^{2}+1}
$$

with $\vartheta=\kappa / \lambda^{2}+\varepsilon, \tilde{h}=h /(1-h)$. The barotropic form stress is thus supported by lateral friction, the baroclinic by interfacial friction and stratification. However, both extract eastward momentum from the flow if the deep transport is eastward: the linear wave model thus does not imply the afore mentioned form stress compensation effect. The total transport $T$ and the shear transport $S$ are readily evaluated as lengthy expressions of $\delta, \varepsilon, \kappa$ and $\lambda$,

$$
T=A W_{0}+B\left(W_{0}+\widetilde{h} Q_{0} / \lambda^{2} \quad h S=C W_{0}+D\left(W_{0}+\widetilde{h} Q_{0} / \lambda^{2}\right)\right.
$$

where the direct wind effect and the baroclinic forcing have been separated. The factors attached to the forcing amplitudes are due to friction and form stress by topographically induced Rossby waves. These transport response functions are

$$
\left(\begin{array}{l}
A \\
B \\
C \\
D
\end{array}\right)=\frac{1}{\varepsilon \vartheta+2(\delta / \beta)^{2} \eta(\vartheta+\widetilde{h} \varepsilon)}\left(\begin{array}{l}
\vartheta+2(\delta / \beta)^{2} \tilde{h} \eta \\
2(\delta / \beta)^{2} \eta \\
2(\delta / \beta)^{2} \widetilde{h} \eta \\
\varepsilon+2(\delta / \beta)^{2} \eta
\end{array}\right)
$$

with $\eta=\varepsilon /\left(1+(\varepsilon / \beta)^{2}\right)+\widetilde{h} \vartheta /\left(1+(\vartheta / \beta)^{2}\right)$ There are three sources of transport according to the relation Eq. (40): $T$ and $h S$ are generated by direct wind forcing, indirect wind forcing via Ekman pumping on the mean stratification, and buoyancy flux. For strictly barotropic conditions where $\lambda \rightarrow 0$ we get $\vartheta \rightarrow \infty$ and the Charney-DeVore result Eq. (22) is
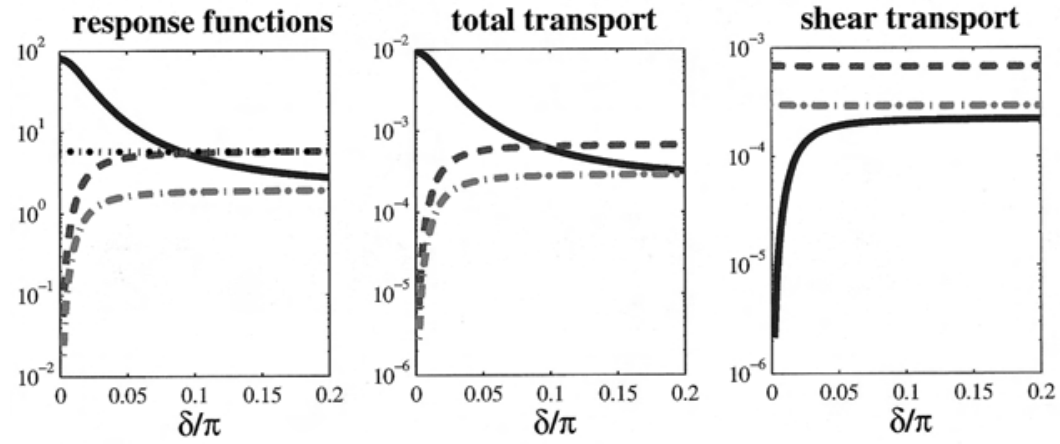

Fig. 22. Left: height dependence of the response functions $A$ [full], $B$ [dashed], $C$ [dashdotted] and $D$ [dotted] of the linear transport model. Middle: the contributions to the total transport $T$ of direct wind forcing [full], Ekman pumping [dashed] and heating [dashdotted]. Right: same for shear transport $h S$. Parameters are: $W_{0}$ corresponds to $0.1 \mathrm{Nm}^{-2}, Q_{0}$ to $10 \mathrm{Wm}^{-2}$ and $\lambda$ to a Rossby radius of $10 \mathrm{~km}$, furthermore $h=0.25, A_{\mathrm{h}}=10^{4} \mathrm{~m}^{2} \mathrm{~s}^{-1}, K$ $=500 \mathrm{~m}^{2} \mathrm{~s}^{-1}$. 


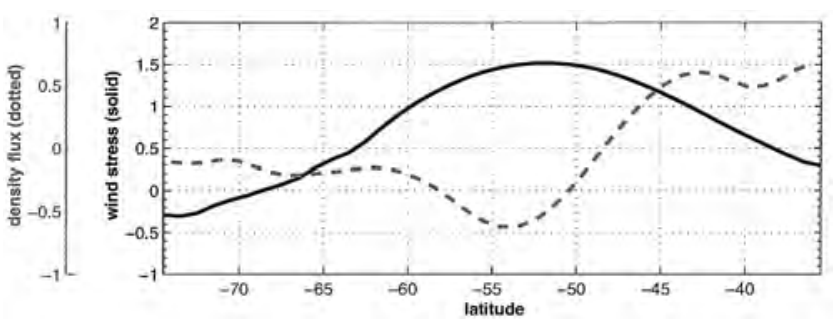

Fig. 23. The forcing data obtained by an ACC-path following average from the NCEP analysis (wind stress: full, units $10^{-4}$ $\mathrm{m}^{2} \mathrm{~s}^{-2}$, density flux: dashed, units $\left.10^{-6} \mathrm{kgm}^{-2} \mathrm{~s}^{-1}\right)$. From Olbers \& Visbeck (2004).

regained.

The response functions $A, B, C$ and $D$ are displayed in Fig. 22 as height dependence for typical parameters. All functions flatten out to a plateau at large topography heights but have different critical heights above which this happens. The critical height of the barotropic response is clearly $\delta \sim \beta$ (see also the Charney-DeVore model Eq. (22)). The baroclinic response scale depends very much on the sizes of $\varepsilon, \kappa$ and $\lambda$. The figure also elucidates the shares of the different transport sources for typical $W_{0}$ and $Q_{0}$ in the total and the shear transports. It becomes clear that only below quite moderate heights of the topography we find the direct wind effect dominating. At larger heights and for the present parameters the Ekman pumping acting on the stratification is the most important driving agent of transport. Only if $\tilde{h} Q_{0} / \lambda^{2}$ becomes of the order of $W_{0}$ the buoyancy forcing is getting effective for the transport.

Finally we consider a nonlinear extension of the above model. We want to improve on the baroclinic bottom form stress and thus still neglect the standing eddy IFS. Including now all terms in the baroclinic wave Eqs (32) \& (34) the baroclinic bottom form stress becomes

$$
\delta h S_{c}=\frac{\vartheta}{(\vartheta / \beta)^{2}+1-\left(n T / \beta \lambda^{2}\right)^{2}}\left(2(\delta / \beta)^{2} \tilde{h}(T-h S)+(\delta / \beta) T_{c} \frac{n h S}{\varepsilon \lambda^{2}}\right)
$$

and it is evident that supercritical conditions, $T>\beta \lambda^{2}$, can indeed lead to a negative baroclinic bottom form stress which then drives the current eastward (if the bottom flow $T-h S$ is eastward and the last term on the rhs is small actually requiring a large viscosity $\varepsilon$ as indicated by the numerical experiments in Fig. 21). We conclude that the supercriticality of the ACC with respect to baroclinic Rossby waves is essential for the system to achieve the observed balance of zonal momentum in which wind and baroclinic bottom form stress drive and barotropic form stress decelerates the current.

\section{The meridional overturning}

Much of the recent perception of the ACC circulation is centered on the meridional overturning and ventilation of water masses in the Southern Ocean. The classical view (Sverdrup et al. 1942) of water mass storage and spreading is sketched in Fig. 4 (Gordon 1999) and repeated in Fig. 12 where the role of eddies in the unblocked part of the watercolumn is highlighted. We have pointed out at various places in this paper that eddies and turbulent mixing might accomplish a major task in shaping and balancing the overturning circulation and it remains to put up a simple strawman model to demonstrate how it might work.

We follow the concept presented in Olbers \& Visbeck (2004) which extends the work of Marshall \& Radko (2003) to a predictive model of the overturning. We assume that all mixing and watermass formation processes take place in an upper layer of the ocean - basically a turbulent layer where
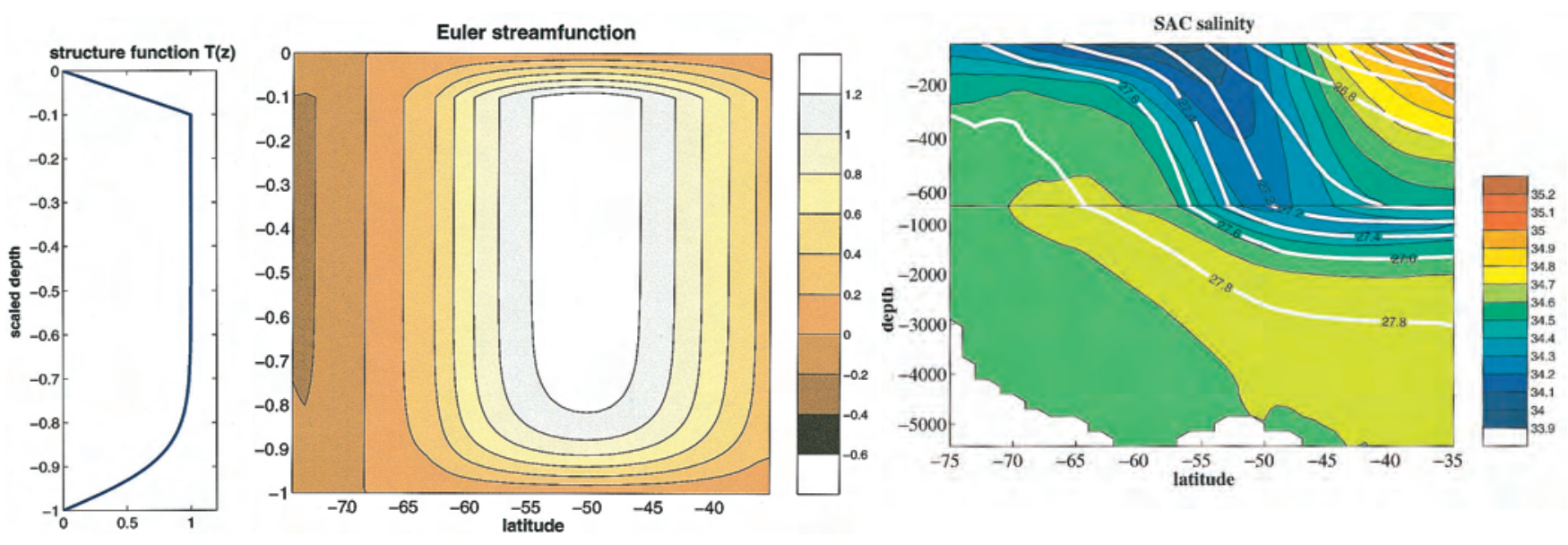

Fig. 24. Left: sketch of the structure function $T(z)$. The depth $z=-d$ marks the base of the upper frictional boundary layer; $z=-h$ is the bottom (maximum depth along circumpolar path), and below $z=-D$ the watercolumn is blocked by topography. Middle: the Euler streamfunction for the average wind stress from Fig. 23 and the structure function $T(z)$. Right: the averaged potential density [white contours] on top of the salinity (ACC-path following average, computed from the SAC climatology). According to the eddy-adiabatic model presented here, the isopycnals coincide with the residual streamfunction pattern. From Olbers \& Visbeck (2004). 

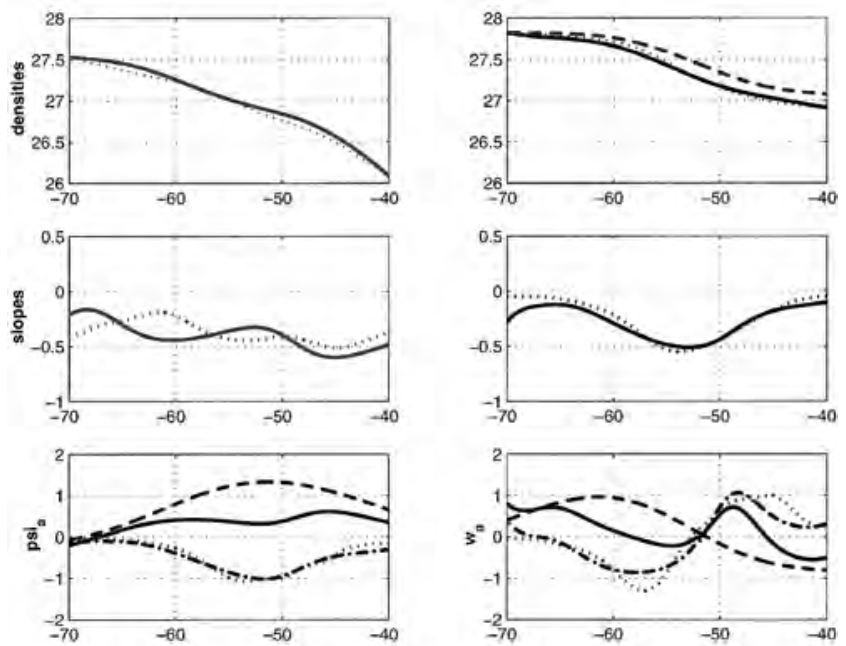

Fig. 25. Solution for parameter values $K_{s}=1000 \mathrm{~m}^{2} \mathrm{~s}^{-1}$ at the southern boundary, linearly increasing by a factor 5 towards the northern side, $\alpha_{d}=8.44 \times 10^{-7} \mathrm{~ms}^{-1}, \alpha_{a}=3 \times 10^{-6} \mathrm{~ms}^{-1}, d=100$ $\mathrm{m}, a=500 \mathrm{~m}$. The upper two panels display the resulting density fields $B_{d}$ and $B_{a}$ [both full] and the middle panels show the slopes [both full]. They are compared to the SAC climatology [dotted]. The deep $\widetilde{B}$ density is dashed [upper right panel]. The lower two panels show the streamfunctions and pumping velocities at the shear layer base $z=-a$ : Ekman streamfunction [dashed], eddy streamfunction [dashdotted], residual streamfunction [full], eddy streamfunction from SAC climatology [dotted], same for the pumping velocities. From Olbers \& Visbeck (2004).

Ekman transport and pumping is established by the wind and buoyancy is imprinted on the surface waters by heat and freshwater flux from the overlying atmosphere - while the ocean interior is void of turbulence but eddies are present that transport and mix substances along isopycnals. We

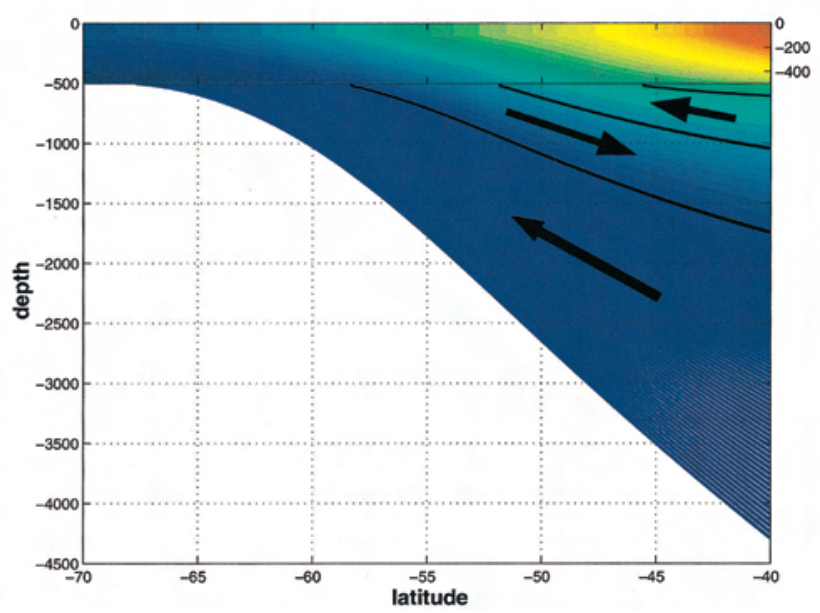

refer to this concept as 'adiabatic eddy regime' and see it as an extreme scenario. The real ocean might have substantial mixing by turbulence in the interior as well (see Heywood et al. 2002, Naveira Garabato et al. 2004). Eddies might contribute by a diapycnal flux to watermass formation, too. However, the above simplified view allows for an analytical treatment. We choose set-up where the equations are averaged along a mean ACC path. We thus neglect the standing eddy component and use the TEM model derived in Section 4.1 with a downgradient parameterization of the meridional density flux by transient eddies, $\left\{v^{\prime} \rho^{\prime}\right\}=-K L B_{y}$. This casts the interior eddy streamfunction into $\phi_{e d}=K s$ where $s=-B_{y} / B_{z}$ is the isopycnal slope. We neglect the eddy Reynolds stress in (26) and assume that the frictional stress $\tau$ acts as a body force which is nonzero only in the surface Ekman layer. Since wind stress and bottom form stress must balance, as discussed in Section 3.3, the amplitude of the form stress is that of the wind stress. Consequently, the Eulerian streamfunction is set by the wind stress and, abbreviating the Ekman transport by $M=-\left\{\tau_{o}\right\} /(L f)$, we finally get $\phi=M T(z)$ and $\phi_{\text {res }}=M T(z)+K s$ where $T(z)$ is a structure function as sketched in Fig. 24.

With no turbulence in the interior we have $\boldsymbol{J} \equiv 0$ and the density budget of (26) becomes $J\left(\phi_{\text {res }}, B\right)=0$, saying that residual streamlines and isopycnals coincide. This can be written as a differential equation for the slope,

$$
\frac{\partial}{\partial y}(K s+M)+s \frac{\partial}{\partial z} K s=0
$$

The isopycnals are the characteristics of this equation. Initial data of $s$ are required on some non-isopycnal curve, in our case this will be the depth level $z=-a$ below which turbulence stops acting. Because $s$ is infinite in the mixed layer where $B=B_{d}(y)$ we assume between the mixed layer

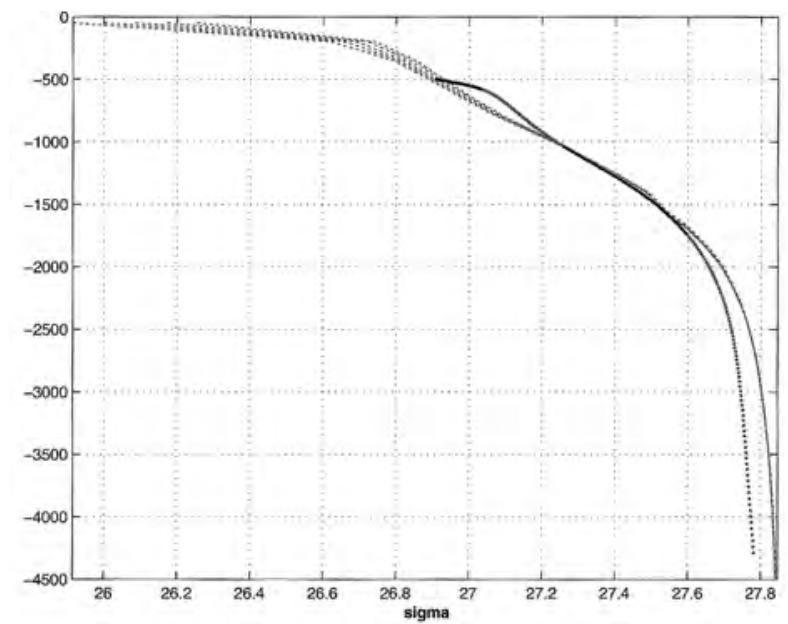

Fig. 26. Left panel: the density field for the upper layer simulation of Fig. 25 . The eddy diffusivity is $K_{i}=500 \mathrm{~m}^{2} \mathrm{~s}^{-1}$ at the southern boundary, linearly increasing by a factor 5 towards the northern side and vertically constant. For the upper $500 \mathrm{~m}$ the solutions $B_{a}$ and $B_{a}$ have been converted to contours, below the characteristics are coloured according to the corresponding density. At black characteristics upward and downward motion changes direction. Right panel: the resulting density values on the northern boundary at $-40^{\circ} \mathrm{S}$ [black and grey curve; grey is upwelling] in comparison to the SAC densities [data from $42^{\circ} \mathrm{S}$ to $38^{\circ} \mathrm{S}$; dashed profiles]. From Olbers \& Visbeck (2004). 


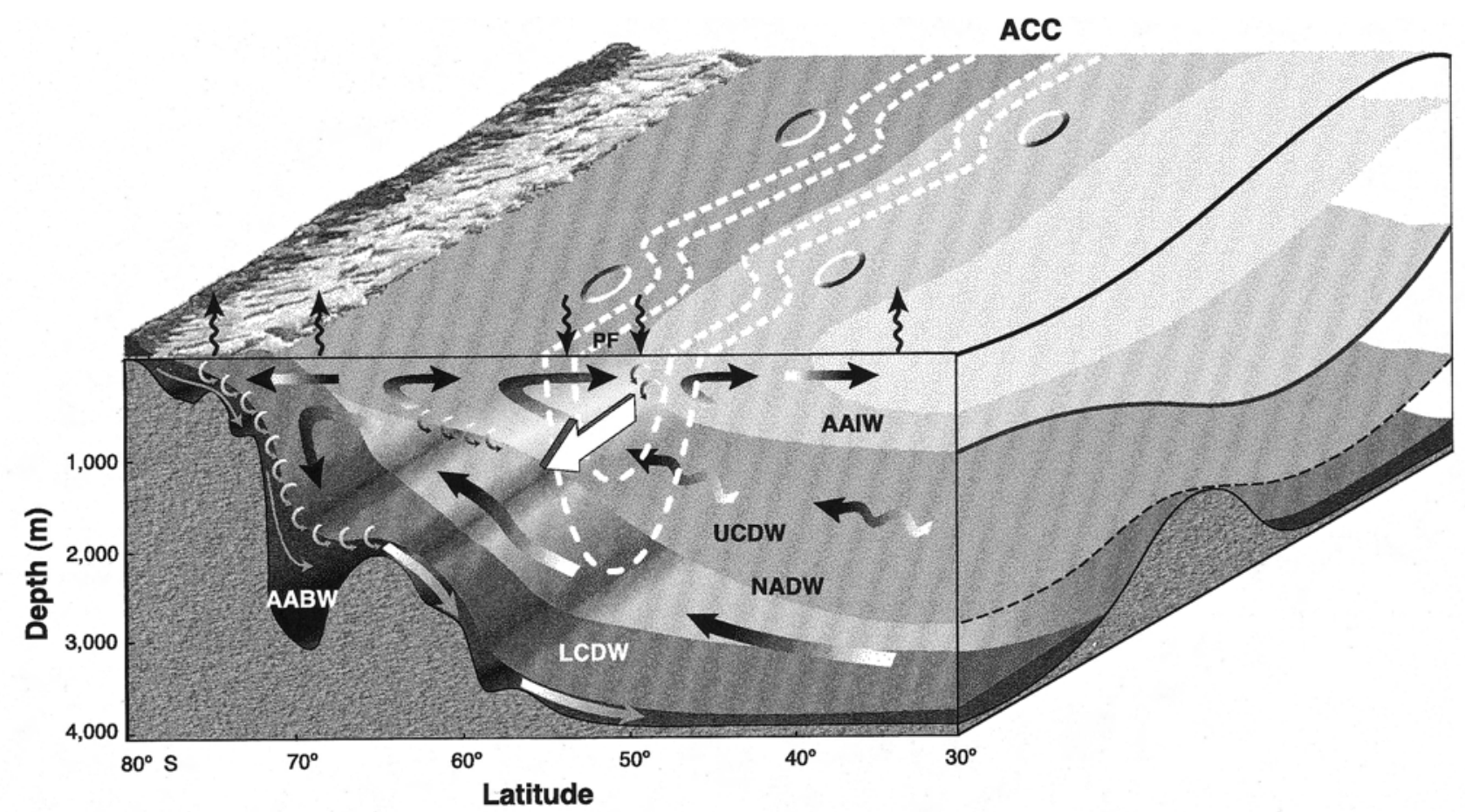

Fig. 27. A sketch of the ACC system showing the zonal flow and the meridional overturning circulation and watermasses. Antarctica is at the left side. The east-west section displays the isopycnal and sea surface tilts in relation to submarine ridges, which are necessary to support the bottom form stress signatures discussed in the text. The curly arrows at the surface indicate the buoyancy flux, the arrows attached to the isopycnals represent turbulent mixing. Sinking of Antarctic Intermediate Water (AAIW) is not shown (see Fig. 24). Redrawn using a figure from Speer et al. (2000).

base $z=-d$ and the depth $z=-a$ an intermediate layer with finite slopes. As in the mixed layer we implement a prescribed structure of the density field in the 'slope layer' $-d \leq z \leq-a$,

$$
B(y, z)=B_{d}(y) \frac{z+a}{a-d}-B_{a}(y) \frac{z+d}{a-d}
$$

The slope at $z=-a$ is then $s_{a}=(a-d)\left(\partial B_{a} / \partial y\right) /\left(B_{a}-B_{d}\right)$.

It remains thus to determine the upper layer densities $B_{d}(y)$ and $B_{a}(y)$. In this depth range the complete nonadiabatic density balance Eq. (23) must be applied. We insert the density structure (mixed layer and slope layer) and deduce a coupled advective-diffusive set of balance equations,

$$
\begin{aligned}
\frac{\partial}{\partial y}\left(K_{m} \frac{\partial B_{d}}{\partial y}\right) & =-\mathcal{H}_{\mathrm{O}}+\left(M+E_{d}\right) \frac{\partial B_{d}}{\partial y}-\alpha_{d}\left(B_{a}-B_{d}\right) \\
\frac{\partial}{\partial y}\left(K_{a} \frac{\partial B_{a}}{\partial y}\right)+\frac{\partial}{\partial y}\left(K_{d} \frac{\partial B_{d}}{\partial y}\right) & =-\frac{\partial M}{\partial y}\left(B_{a}-B_{d}\right)+E_{a} \frac{\partial B_{a}}{\partial y}-E_{d} \frac{\partial B_{d}}{\partial y}+ \\
& +\alpha_{d}\left(B_{a}-B_{d}\right)+\alpha_{a}\left(B_{a}-\widetilde{B}\right)
\end{aligned}
$$

Advection by the eddy streamfunction enters as $E_{d}=\phi_{e d}$ $(z=-d)=K(z=-d) s_{d}$ and $E_{a}=\phi_{e d}(z=-a)=K(z=-a) s_{a}$. Apart from advection eddies also lead to diffusion of density: the diffusivities on the lhs are vertical integrals of $K(y, z)$. The equations describe our basic perception of the processes that happen in the upper layer. The mixed layer density is forced by the surface density flux $\mathcal{H}_{0}$ from exchange by heat and freshwater with the atmosphere (we use the forcing data shown in Fig. 23). There is meridional advection by the Eulerian Ekman currents and by eddies, there is vertical pumping by these agents (eddy pumping would appear if the advection terms are split into complete divergences and vertical advection), with mixing by turbulence to sustain the vertically mixed state $B_{d}(y)$, and entrainment of density at the mixed layer base, parameterized by a mixing coefficient $\alpha_{d}$. Corresponding terms arise in the slope layer balance. The density $\widetilde{B}$ that is entrained into the slope layer from below is the density at depth ranges with inward flow at the northern boundary of the model domain, e.g. NADW at $40^{\circ} \mathrm{S}$. This water proceeds up the isopycnals to the slope layer base without changing its density. The coupling to the interior ocean occurs via the predicted slopes at the base of the slope layer.

The performance of the model is exemplified in Figs 25 \& 26 , using reasonable parameter values (see figure caption). It should, however, be borne in mind that the solution structure might change substantially if another choice is made, in particular in the interior where the slopes are inversely proportional to the value of the eddy diffusivity $K$ and changes at some level influence via the characteristics the entire deeper structure. For the simulation shown in Fig. 26 we have used the most simple form oriented at the diffusivity estimates discussed in Olbers \& Visbeck (2004): 
$K(y, z)$ is vertically constant and a linear function of $y$ with significant increase (by a factor of 5) towards the north. This particular solution yields an up-down-up-down pattern of pumping (by eddies and Ekman) at the slope layer base (see the solid curve in the lower right panel of Fig. 25) which in this structure may be associated with upwelling NADW, downwelling AAIW and upwelling Subantarctic mode water (the latitudes and densities are roughly consistent with this interpretation). Propagation of the upper layer solution via the characteristic Eq. (43) into the interior yields the associated isopycnals (and residual streamfunction) which also coincide well with the observed interior density structure (see Fig. 24 and the left panel of Fig. 26). We would like to point out that the eddy field has a dominant share in this simulation: without eddies the streamfunction and densities would mirror the Deacon cell depicted in Fig. 24. This is also evident from the streamfunctions and associated pumping velocities of the solution shown in the lower panels of Fig. 25.

\section{Conclusions}

There are five threads running through this review of the ACC system: submarine topography, standing and transient eddies, long barotropic and baroclinic Rossby waves, turbulent mixing and surface flux of momentum and buoyancy. They are woven into the physics governing this extraordinary current, attempting to contribute to an answer of the most interesting and most urgent questions about the ACC: what is the balance of the zonal momentum; what mechanism and forcing functions determine the transport; how do watermasses and the substances they carry penetrate the strong and deep-reaching zonal flow? We base our discussion on the research on these topics which has accumulated in the last decade and partly answer these questions. Our tools are observations, theory and models coming from a variety of instrumental techniques, field expeditions and modelling concepts. Fig. 27 gives a sketch of the physics in the ACC system showing its most important ingredients.

The flow achieves a balance of its zonal momentum in which the input of eastward momentum from the wind stress acting all the way around Antarctica is transferred by standing and transient eddies through the water column to the bottom where the bottom pressure field adjusts such that bottom form stress acts as a sink. Clearly, if adjustment is not present, the imbalance will accelerate the current, inducing changes of the pressure field until a balance has been reached. Separating this form stress into the one arising from the surface pressure and the one due to the internal mass stratification it is seen that these individual components overwhelm the wind stress by an order of magnitude, the barotropic form stress is retarding the eastward current and the baroclinic is accelerating it (see east-west interface of Fig. 27). Both, however, must compensate to a high degree, a constraint that arises from the balance of the zonal mean isopycnal thickness structure where vertical pumping by the Ekman, barotropic and baroclinic velocities of the current acting on the mean stratification are dominating and must cancel each other. The large scale vertical flow comes about by the zonal flow passing across the large-scale topographic barriers, mainly the midocean ridges along the path of the ACC in the Southern Ocean. We have found evidence that the driving of the eastward current by the baroclinic bottom form stress needs a supercriticallity of the flow with respect to the associated long planetary Rossby waves. They must be advected eastward by the current to become locked with the required phase shift to the zonal topographic undulations.

The downward transport of zonal momentum is established by the mechanism of interfacial form stress by which pressure forces act across the isopycnal interfaces which are tilted by the action of transient and standing eddies. The form stress is associated with a meridional eddy density (or heat) flux, and a downward flux of eastward momentum supports a poleward heat flux, in agreement with observations and eddy-resolving models. The vertical divergence of interfacial form stress drives the meridional overturning circulation. In isopycnal ranges which are zonally unblocked by submarine topography the divergence of the interfacial form stress is the dominant driving force of the meridional flow - it is eddy-driven as indicated by the wavy arrows at intermediate depths on the frontside of Fig. 27. Below in the blocked range of isopycnals we have geostrophic meridional flow in the valleys between the submarine ridges, these are supported by the pressure gradients associated with the bottom form stress. And above the eddy-driven regime we find the northward Ekman flow which is driven by the eastward zonal wind. Clearly, the balance of momentum and the forces driving the meridional overturning circulation correspond to each other (in a mathematical frame they are described by the same equations).

The isopycnals in the Southern Ocean connect the deep ocean to the north of the ACC to the surface areas to the south, the ACC being attached to stronger tilts correlated over the depth. We have turned existing concepts on processes shaping the isopycnal stack in the Southern Ocean into a prognostic theory. Also for these processes we refer to Fig. 27. The Eulerian mean flow and the eddies combine to transport density (heat and substances) to and from the upper ocean layer where mixing by small-scale turbulence and exchange of heat and freshwater with the atmosphere must occur. Two assumptions imply that the mean transport in the interior - below the mixed layer - by the mean flow and eddies is entirely along the isopycnals. These are: eddies do not carry stuff across isopycnals, only along them; diapycnal mixing by small-scale turbulence is absent below the mixed layer. Then streamlines of transport by mean flow and eddies, representing the residual 
circulation, coincide with isopycnals. The concept allows the complicated mathematics of an advection-diffusionmixing regime to be broken into manageable parts - mixed layer physics with Ekman and eddy advection and a diabatic interior - which may be solved by simpler means. We are able to predict the density field - the decrease of surface density with increasing latitude and the shape of the downward sloping isopycnals - from the wind field and buoyancy flux through the ocean surface. The theory applies to an ACC path following average and thus is rather qualitative but it demonstrates the overwhelming importance of the transient eddy field in shaping the density field in the Southern Ocean.

The last concern of our review is the issue of transport of the ACC. Over many decades the current was considered basically wind-driven and its transport attributed to the direct action of the wind over the Southern Ocean as the most prominent driving agent, though early experiments with global OGCMs clearly showed the importance of baroclinicity on the ACC transport. A more detailed transport theory is recapitulated below in two steps.

If a mean stratification is considered as a given horizontally uniform background (a mean Brunt-Vaisala frequency profile or an isopycnal layer stack) the effect of the wind stress is not limited to a direct driving of currents by friction but there is in addition the Ekman pumping acting to deform the stratification. Together with a prescribed input of buoyancy (say, cooling in the south and heating in the north) this Ekman pumping sets up a baroclinic pressure force which gives rise to a baroclinic component of the current. Feedback by bottom form stress (the Rossby wave connection mentioned above) directly influences the zonal transport. In summary, the ACC transport has a direct contribution from the wind stress, an 'indirect' contribution from the Ekman pumping on the mean stratification, and a contribution from the prescribed surface buoyancy flux. The latter two contributions only appear if the topography is undulated (if bottom form stress may act). Our simple linear transport model elucidates these mechanisms and clearly shows that for sufficiently high topography amplitudes the indirect wind forcing and the external heating may be dominant over the direct wind effect.

In a second stage we realize that the stratification is not given but depends on many processes, among them also features of the local wind stress and surface buoyancy flux not considered so far. Non-zonal pattern in these fluxes (e.g. more cooling in the Atlantic sector of the Southern Ocean than elsewhere) may generate zonal pressure forces in the bottom form stress and influence the transport. There is also the possibility of remote control via the NADW/conveyor belt connection. There are other second order effects such as the influence of the winds on turbulence and mixing and regional differences in the transient eddy energy. We did not attempt to quantify these effects on the ACC transport.
The concepts and models discussed in this review would greatly benefit from extended knowledge in some specific areas. From the experimental and field work community we need information about the level and regional distribution of diapycnal mixing in the area south of the ACC, in particular where in the water column it occurs predominantly. Furthermore we would profit from better knowledge about the parametrization of isopycnal eddy fluxes which certainly needs a combination of theoretical work and eddyresolving modelling efforts in idealized and realistic configurations. Finally, we must admit that many of the considerations in this review are based on relatively simple models and model diagnostics. We certainly would profit from improved diagnostic utilization of realistic eddyresolving models toward an understanding of mechanisms outlined in this article, e.g. the establishment of form stress in the interior and at the bottom by waves and eddies.

\section{Acknowledgements}

We appreciate numerous discussions with Carsten Eden and Sergey Danilov and are grateful for the extensive and helpful comments of three referees. We greatly benefited from Stephen Rintoul's comments on the manuscript.

\section{References}

ANDERSON, D.L.T. \& GiLl, A.E. 1975. Spin-up of a stratified ocean, with applications to upwelling. Deep-Sea Research, 22, 583-586.

Andrews, D.G., Holton, J.R. \& Leovy, C.B. 1987. Middle atmosphere dynamics. London: Academic Press, 489 pp.

BAKER JR, D.J. 1982. A note on Sverdrup balance in the Southern Ocean. Journal of Marine Research, 40 (Suppl.), 21-26.

BERgER, W.H. 1981. Paleoceanography: the deep-sea record. In EMILIANI, C., ed. The oceanic lithosphere; the sea, vol. 7. New York. WileyInterscience, 1437-1519.

Best, S.E., Ivchenko, V.O., Richards, K.J., Smith, R.D. \& Malone, R.C. 1999. Eddies in numerical models of the Antarctic Circumpolar Current and their influence on the mean flow. Journal of Physical Oceanography, 29, 328-350.

Borowski, D. 2003. The Antarctic Circumpolar Current: dynamics of a circumpolar channel with blocked geostrophic contours. $\mathrm{PhD}$ thesis, University Bremen, Germany. [Unpublished].

Borowski, D., Gerdes, R. \& Olbers, D. 2002. Thermohaline and wind forcing of a circumpolar channel with blocked geostrophic contours. Journal of Physical Oceanography, 32, 2520-2540.

BROECKER, W.S. 1991. The great ocean conveyor. Oceanography, 4, 79-89.

BRYAN, F.O. 1997. The axial angular momentum balance of a global ocean general circulation model. Dynamics of Atmosphere and Oceans, 25, 191-216.

BRYAN, K. \& Cox, M.D. 1972. The circulation of the World Ocean: a numerical study. Part I, A homogeneous model. Journal of Physical Oceanography, 2, 319-335.

BRYAN, K., Dukowicz, J.K. \& SMith, R.D. 1999. On the mixing coefficient in the parameterization of bolus velocity. Journal of Physical Oceanography, 29, 2442-2456.

BRYDEN, H.L. \& CunNingHAM, S.A. 2003. How wind-forcing and air-sea heat exchange determine the meridional temperature gradient and stratification for the Antarctic Circumpolar Current. Journal of Geophysical Research, 108, doi:10.1029/2001JC0011296. 
Bryden, H.L. \& Heath, R.A. 1985. Energetic eddies at the northern edge of the Antarctic Circumpolar Current in the South Pacific. Progress in Oceanography, 14, 65-87.

CAI, W. \& BAINes, P.G. 1996. Interactions between thermohaline- and wind-driven circulations and their relevance to the dynamics of the Antarctic Circumpolar Current, in an coarse-resolution global ocean general circulation model. Journal of Geophysical Research, 101, 14073-14093.

Charney, J.G. \& DeVore, J.G. 1979. Multiple flow equilibra in the atmosphere and blocking. Journal of Atmospheric Sciences, 36, $1205-1216$

COWARD, A.C. 1996. Current status of the OCCAM global ocean model. Sigma (UK WOCE Newsletter; unpublished manuscript), 20, 4-6.

Cox, M.D. 1975. A baroclinic numerical model of the Word Ocean: preliminary results. In Numerical Models of Ocean Circulation. Durham, New Hampshire, Oct. 17-20, 1972. 107-120

Cunningham, S., Alderson, S., King, B.A. \& Brandon, M.A. 2003. Transport and variability of the Antarctic Circumpolar Current in Drake Passage. Journal of Geophysical Research, 108, doi: 10.1029/2001JC001147.

Danabasoglu, G. \& McWilliams, J.C. 1995. Sensitivity of the global ocean circulation to parameterization of mesoscale tracer transports. Journal of Climate, 8, 2967-2987.

DeSzoeke, R.A. \& Levine, M.D. 1981. The advective flux of heat by mean geostrophic motions in the Southern Ocean. Deep-Sea Research, 28, $1057-1085$.

DiJKstra, H.A. 2000. Nonlinear physical oceanography. Dordrecht: Kluwer Academic Publishers, 480 pp.

Döös, K. \& WebB, D.J. 1994. The Deacon cell and other meridional cells of the Southern Ocean. Journal of Physical Oceanography, 24, $429-442$.

EADY, E.T. 1949. Long waves and cyclone waves. Tellus, 1, 33-52.

FAHrbach, E., SchröDER, M. \& KlepiKov, A. 1994. Circulation and water masses in the Weddell Sea. Technical report, Physics of ice-covered seas: Lecture Notes from a Summer School, Savonlinna, Finland, Helsinki University Press, Helsinki, 2, 569-604.

Fox, A.D. Haines, K., De Cuevas, B.A. \& WebB, D.J. 2000. Altimeter assimilation in the OCCAM global model - Part I: A twin experiment. Journal of Marine Systems, 26, 303-322.

FRAM GROUP. 1991. An eddy-resolving model of the Southern Ocean. EOS, 72 (15), 169-175.

Fritzsch, B., Gerdes, R., Hiller, W., Latif, M., Legutke, S., MaierReimer, E., Olbers, D. \& Roeske, F. 2000. Vergleich der thermohalinen Zirkulation in zwei globalen ozeanischen Zirkulationsmodellen - The Ocean Model Intercomparison Project. Abschlubericht BMBF Projekt 01 LA 9862/8, 133 pp, unpublished manuscript.

Gent, P.R. \& McWilliams, J.C. 1990. Isopycnal mixing in ocean circulation models. Journal of Physical Oceanography, 20, 150-155.

Gent, P.R., Large, W.G. \& Bryan, F.O. 2001. What sets the mean transport through Drake Passage? Journal of Geophysical Research, 106, 2693-2712.

Gent, P.R., Willebrand, J., McDougall, T.J. \& McWilliams, J.C. 1995. Parameterizing eddy-induced tracer transports in ocean circulation models. Journal of Physical Oceanography, 25, 463-474.

GILL, A.E. 1968. A linear model of the Antarctic Circumpolar Current. Journal of Fluid Mechanics, 32, 465-488.

GiLLE, S.T. 1994. Mean sea surface height of the Antarctic Circumpolar Current from Geosat data: Method and application. Journal of Geophysical Research, 99, 18 255-18 274.

GiLLE, S.T. 2003. Float observations of the Southern Ocean. Part II: Eddy fluxes. Journal of Physical Oceanography, 33, 1182-1196.

Gille, S.T., Yale, M.M. \& SAndwell, D.T. 2000. Global correlation of mesoscale ocean variability with seafloor roughness from satellite altimetry. Geophysical Research Letters, 27, 1251-1254.
Gnanadesikan, A. \& Hallberg, R.W. 2000. On the relationship of the Circumpolar Current to Southern Hemisphere winds in coarse resolution ocean models. Journal of Physical Oceanography, 30, 2013-2034.

GoRDON, A.L. 1986. Interocean exchange of thermocline water. Journal of Geophysical Research, 91, 5037-5046.

Gordon, A.L. 1999. The Southern Ocean currents. Journal of Marine Education, 15, 4-6.

Green, J.S.A. 1970. Transfer properties of the large-scale eddies and the general circulation of the atmosphere. Quarterly Journal of the Royal Meteorological Society, 96, 157-185.

HAN, Y.-J. 1984a. A numerical world ocean general circulation model. Part I. Basic design and barotropic experiment. Dynamics of Atmosphere and Oceans, 8, 107-140.

HAN, Y.-J. 1984b. A numerical world ocean general circulation model. Part II. A baroclinic experiment. Dynamics of Atmosphere and Oceans, $\mathbf{8}$, 141-172.

Heywood, K., Garabato, A.C.N. \& Stevens, D.P. 2002. High mixing rates in the abyssal Southrn Ocean. Nature, 415, 1011-1014.

HidAKA, K. \& TsuchiYA, M. 1953. On the Antarctic Circumpolar Current. Journal of Marine Research, 12, 214-222.

Holland, W.R. 1973. Baroclinic and topographic influences on the transport in western boundary currents. Geophysical Fluid Dynamics, $\mathbf{4}$, $187-210$.

Houry, S., Dombrowsky, E., De Mey, P. \& Minster, J.-F. 1987. BruntVaisala frequency and Rossby radii in the Southern Ocean. Journal of Physical Oceanography, 17, 1619-1626.

Hughes, C.W. 2002. Sverdrup-like theories of the Antarctic Circumpolar Current. Journal of Marine Research, 60, 1-17.

Hughes, C.W. \& De Cuevas, B.A. 2001. Why western boundary currents in realistic oceans are inviscid: a link between form stress and bottom pressure torques. Journal of Physical Oceanography, 31, 2871-2885.

Hughes, C.W. \& Ash, E.R. 2001. Eddy forcing of the mean flow in the Southern Ocean. Journal of Geophysical Research, 106, 2713-2722.

Hughes, C.W., Jones, M.S. \& Carnochan, S. 1998. Use of transient features to identify eastward currents in the Southern Ocean. Journal of Geophysical Research, 103, 2929-2944.

IsHIDA, A. 1994. Effects of partial meridional barriers on the Antarctic Circumpolar Current wind-driven barotropic model. Dynamics of Atmosphere and Oceans, 20, 315-341.

IvchenKo, V.O., Richards, K.J. \& SteVEns, D.P. 1998. The dynamics of the Antarctic Circumpolar Current. Journal of Physical Oceanography, 26, 753-774.

Johnson, G.C. \& Bryden, H.L. 1989. On the size of the Antarctic Circumpolar Current. Deep-Sea Research, 36, 39-53.

Josey, S.A., Kent, E.C. \& TAYLOR, P.K. 1998. The Southampton Oceanography Centre (SOC) ocean-atmosphere heat, momentum and freshwater flux atlas. Technical report, Southampton Oceanography Centre, Report No. 6., Southampton, United Kingdom, 30 pp.

Karsten, R.H., Jones, H. \& Marshall, J. 2002. The role of eddy transfer in setting the stratification and transport of a Circumpolar Current. Journal of Physical Oceanography, 32, 39-54.

Karsten, R.H. \& Marshall, J. 2002. Testing theories of the vertical stratification of the ACC against observations. Dynamics of Atmosphere and Oceans, 36, 233-246.

Killworth, P.D. \&. NANNEH, M.M 1994. Isopycnal momentum budget of the Antarctic Circumpolar Current in the Fine Resolution Antarctic Model. Journal of Physical Oceanography, 24, 1201-1223.

KRUPitsky, A. \& CANE, M.A. 1994. On topographic pressure drag in a zonal channel. Journal of Marine Research, 52, 1-23.

Maltrud, M.E., Smith, R.D., Semtner, A.J. \& Malone, R.C. 1998. Global eddy-resolving ocean simulations driven by 1985-1994 atmospheric winds. Journal of Geophysical Research, 103, $30825-30853$.

Marshall, J. \& RADKo, T. 2003. Residual-mean solutions for the Antarctic Circumpolar Current and its associated overturning circulation. Journal of Physical Oceanography, 33, 2341-2354. 
Marshall, J., Olbers, D., Ross, H. \& Wolf-Gladrow, D. 1993. Potential vorticity constraints on the dynamics and hydrography in the Southern Ocean. Journal of Physical Oceanography, 23, 465-487.

McIntosh, P.C. \& McDougall, T.J. 1996. Isopycnic averaging and the residual mean circulation. Journal of Physical Oceanography, 26, $1656-1660$.

Morrow, R., Coleman, R., Church, J. \& Chelton, D. 1994. Surface eddy momentum flux and velocity variances in the southern ocean from geosat altimetry. Journal of Physical Oceanography, 24, 2050-2071.

MunK, W.H. \& Palmen, E. 1951. Note on dynamics of the Antarctic Circumpolar Current. Tellus, 3, 53-55.

Naveira Garabato, A.C., Polzin, K.L., King, B.A., Heywood, K.J. \& VISBECK, M. 2004. Widespread intense turbulent mixing in the Southern Ocean. Science, 303, 210-213.

Nowlin JR, W.D., Worley, S.J. \& Whitworth III, T. 1985. Methods for making point estimates of eddy heat flux as applied to the Antarctic Circumpolar Current. Journal of Geophysical Research, 90, 3305-3324.

OlBERs, D. 1998. Comment on 'On the obscurantist physics of 'form drag' in theorizing about the Circumpolar Current. Journal of Physical Oceanography, 28, 1647-1654.

Olbers, D. 2001. A gallery of simple models from climate physics. In ImKeller, P. \& von Storch, J., eds. Stochastic climate models. Basel: Birkhauser, 3-63.

OlBERs, D. 2005. On the role of eddy mixing on the transport of zonal ocean currents. In Baumert, H., Simpson, J. \& SÜndermann, J., eds. Marine turbulence: theories, observations and models. Cambridge: Cambridge University Press, 511-529.

OlBERs, D \& EDEN, C. 2003. A simplified general circulation model for a baroclinic ocean with topography. Part I, Theory, waves and winddriven circulations. Journal of Physical Oceanography, 33, 2719-2737.

OlBERS, D. \& VölKer, C. 1996. Steady states and variability in oceanic zonal flows. In Anderson, D.L.T. \& Willebrand, J., eds. Decadal climate variability dynamics and prediction. Berlin: Springer, 407-443.

Olbers, D \& VisBeCK, M. 2004. A model of the zonally averaged stratification and overturning in the Southern Ocean. Journal of Physical Oceanography.

OlBers, D. \& IVChENKo, V.O. 2001. On the meridional circulation and balance of momentum in the Southern Ocean of POP. Ocean Dynamics, 52, 79-93.

Olbers, D., Wolff, J.-O. \& VölKer, C. 2000. Eddy fluxes and secondorder moment balances for nonhomogeneous quasigeostrophic turbulence in wind-driven zonal flows. Journal of Physical Oceanography, 30, 1645-1668.

Olbers D., WüBber C. \& WolfF, J.O. 1992. The dynamical balance of wind and buoyancy driven circumpolar currents. Alfred-WegenerInstitut fur Polar- und Meeres-forschung, Bremerhaven, Research Report 32, $47 \mathrm{pp}$.

OlBERS, D.J. \& WÜBBER, C. 1991. The role of wind and buoyancy forcing of the Antarctic Circumpolar Current. In LATIF, M., ed. Strategies for Future Climate Research. Mainz: Max-Planck-Institut fur Meteorologie, 161-191.

Olbers, D.J., Wenzel, M. \& Willebrand, J. 1985. The inference of North Atlantic circulation patterns from climatological hydrographic data. Reviews of Geophysics, 23, 313-356.

Orsi, A.H., Whitworth III, T.W. \& Nowlin JR, W.D. 1995. On the meridional extent and fronts of the Antarctic Circumpolar Current. Deep-Sea Research, 42, 641-673.

Peixoto, J.P. \& Oort, A.H. 1992. Physics of climate. New York: American Institute of Physics, $520 \mathrm{pp}$.

PhiLLIPS, H.E. \& RinToul, S.R. 2000. Eddy variability and energetics from direct current measurements in the Antarctic Circumpolar Current south of Australia. Journal of Physical Oceanography, 30, 3050-3076.
RHINES, P.B. \& Young, W.R. 1982. A theory of the wind-driven circulation I. Mid-ocean gyres. Journal of Marine Research, 40 (Suppl.), 559-596.

Rintoul, S.R., Hughes, C. \& Olbers, D. 2001. The Antarctic Circumpolar Current system. In Siedler, G. Church J. \& Gould, J., eds. Ocean circulation and climate. New York: Academic Press, 271-302.

RinToul, S.R. \& SoKOLOv, S. 2001. Baroclinic transport variability of the Antarctic Circumpolar Current south of Australia (WOCE repeat section SR3). Journal of Geophysical Research, 106, 2815-2832.

Rintoul, S.R., SOKOlov, S. \& CHURCH, J. 2002. A 6 year record of baroclinic transport variability of the Antarctic Circumpolar Current at 140 degrees $\mathrm{E}$ derived from expendable bathythermograph and altimeter measurements. Journal of Geophysical Research, 107, 3155-3177.

SARKISYAN, A.S. \& IVANOV, V.F. 1971. Joint effect of baroclinicity and bottom relief as an important factor in the dynamics of sea currents. Doklady Akademii Nauk SSSR Atmosph. Oceanic Phyics, 7, 173-188.

SCHMITZ JR, W.J. 1995. On the interbasin-scale thermohaline circulation. Review of Geophysics, 33, 151-173.

Semtner, A.J. \& Chervin, R.M. 1992. Ocean general circulation from a global eddy-resolving model. Journal of Geophysical Research, 97, $5493-5550$.

Speer, K., Rintoul, S. \& Sloyan, B. 2000. The diabatic Deacon cell. Journal of Physical Oceanography, 30, 3212-3222.

Stevens, D.P. \& IvchenKo, V.O. 1997. The zonal momentum balance in an eddy-resolving general-circulation model of the Southern Ocean. Quarterly Journal of the Royal Meteorological Society, 123, 929-951.

Stommel, H. 1957. A survey of ocean current theory. Deep-Sea Research, $4,149-184$.

StonE, P.H. 1972. A simplified radiative-dynamical model for the static stability of rotating atmospheres. Journal of Atmospheric Sciences, 29, 405-418.

SVERDRUP, H.U., Johnson, M.W. \& Fleming, R.H. 1942. The oceans: their physics, chemistry and general biology. Englewood Cliffs, NJ: PrenticeHall, $1087 \mathrm{pp}$.

TAnsley, C.E. \& Marshall, D.P. 2001. On the dynamics of wind-driven circumpolar currents. Journal of Physical Oceanography, 31, $3258-3273$.

Visbeck, M., Marshall, J., Haine, T. \& Spall, M. 1997. Specification of eddy transfer coefficients in coarse-resolution ocean circulation models. Journal of Physical Oceanography, 27, 381-402.

VÖLKER, C. 1999. Momentum balance in zonal flows and resonance of baroclinic Rossby waves. Journal of Physical Oceanography, 29, $1666-1681$.

WANG, L. \& HUANG, R.X. 1995. A linear homogeneous model of winddriven circulation in a $\beta$-plane channel. Journal of Physical Oceanography, 25, 587-603.

WeBB, D.J. 1993. A simple model of the effect of the Kerguelen Plateau on the strength of the Antarctic Circumpolar Current. Geophysical and Astrophysical Fluid Dynamics, 70, 57-84.

Wells, N.C. \& De Cuevas, B.A. 1995. Vorticity dynamics of the Southern Ocean from a General Circulation Model. Journal of Physical Oceanography, 25, 2569-2582.

Whitworth III, T. 1983. Monitoring the transport of the Antarctic Circumpolar Current at Drake Passage. Journal of Physical Oceanography, 13, 2045-2057.

Whitworth III, T. \& Peterson, R.G. 1985. Volume transport of the Antarctic Circumpolar Current from bottom pressure measurements. Journal of Physical Oceanography, 15, 810-816.

WolfF, J.-O., Maier-Reimer, E. \& Olbers, D.J. 1991. Wind-driven flow over topography in a zonal $\beta$-plane channel: a quasi-geostrophic model of the Antarctic Circumpolar Current. Journal of Physical Oceanography, 21, 236-264. 